\title{
GRAVITY SURVEY IN THE CENTRAL RANGES, HONSHU, JAPAN
}

\author{
Akihiko Yamamoto, ${ }^{*}$ Kyozo NozakI, **** Yoshio Fukao,* Muneyoshi Furumoto,* \\ Ryuichi SHICHI, ${ }^{* *}$ and Teruya EzAKa*,**** \\ *Department of Earth Sciences, Nagoya University, Nagoya, Japan \\ **Inuyama Crustal Movement Observatory, Nagoya University, Nagoya, Japan \\ (Received July 1, 1981; Revised June 10, 1982)
}

\begin{abstract}
An extensive gravity survey was carried out from 1978 through 1980 in the area of the Central Ranges (Japan Alps), Honshu, which consist of the three largest mountain ranges in Japan (Hida, Akaishi, and Kiso). The Central Ranges area of $40,000 \mathrm{~km}^{2}$ has provided about 3,700 gravity data including 2,214 newly measured data, from which a Bouguer anomaly map was drawn. Bouguer and terrain corrections were made with a topographic data file of $230 \times 280 \mathrm{~m}^{2}$ grid at a distance of up to $80 \mathrm{~km}$ from any measuring point, taking the earth's sphericity into account. A value of $2.64 \mathrm{~g} / \mathrm{cm}^{3}$ was used. for the average density of surface features, and this was derived from the observed gravity data using a newly proposed least-squares method. The possible errors involved in the analysis were critically evaluated. The newly drawn anomaly map has an overall accuracy of $5 \mathrm{mgal}\left(=5 \times 10^{-5} \mathrm{~m} \cdot \mathrm{sec}^{-2}\right)$. The Central Ranges area is known as a broad zone of strong negative anomaly, which has now been resolved into two parts, one along the axis of the Hida mountain range and the other along the northern Fossa Magna. Bouguer anomaly and topography are correlated negatively in the Hida range, marginally in the Akaishi range and positively in the Kiso range, suggesting that the correlation becomes progressively negative with increasing massiveness of mountain structure. The Central Ranges are bounded by the Itoigawa-Shizuoka Tectonic Line to the east and by the Atera fault to the southwest across which the regional Bouguer anomaly pattern changes sharply. The Atera fault is a firstclass active fault along which a narrow but very clear Bouguer low is observed that may be attributable to fault gouge material.
\end{abstract}

\section{Introduction}

The land gravity data so far obtained through nation-wide measurements in Japan have been summarized by Tsubor et al. (1955), the GEOGRAPHICAL SuRVEY INSTITUTE (GSI) (1964) and HagIwARA (1967). Rather local gravity surveys have been carried out in Central Honshu by the Geological Survey of Japan

\footnotetext{
*** Now with OYO Co. Urawa Research Institute.

**** Now with Japan Petroleum Exploration Co., Ltd.
} 
(GSJ) (e.g., CHujo and SudA, 1972) and other institutions. However, the gravity data available in and around the Central Ranges (Japan Alps) comprising the highest mountain ranges in Japan are very few, as the past measurements were restricted mainly to the first and second order bench marks and triangulation stations along the major national routes. In 1978-1980 we performed an extensive gravity survey in the area of the Central Ranges to obtain a detailed Bouguer anomaly map. A part of the results has already been reported (NoZAKI, 1980). This paper describes the procedure of the survey, the method of analysis and several outstanding features of the new anomaly map. A full discussion of the Bouguer anomaly distribution will be made in a separate paper.

\section{Outline of the Area of Survey}

Figure 1 shows the area of our survey, which is located at the junction of the northeastern Honshu arc, the southwestern Honshu arc and the Izu-Bonin arc. All the mountains in Japan more than 3,000 m high, except for Mt. Fuji, gather in the central portion of this area. This portion is called the Central Ranges or the Japan Alps, comprising three mountain ranges (Hida, Kiso, and Akaishi) that align in the NNW-SSE direction in echelon, each trending approximately in the NNE-SSW direction (see Fig. 9). This trend is perpendicular to the inferred orientation of horizontal compression now causing intraplate earthquakes in Japan

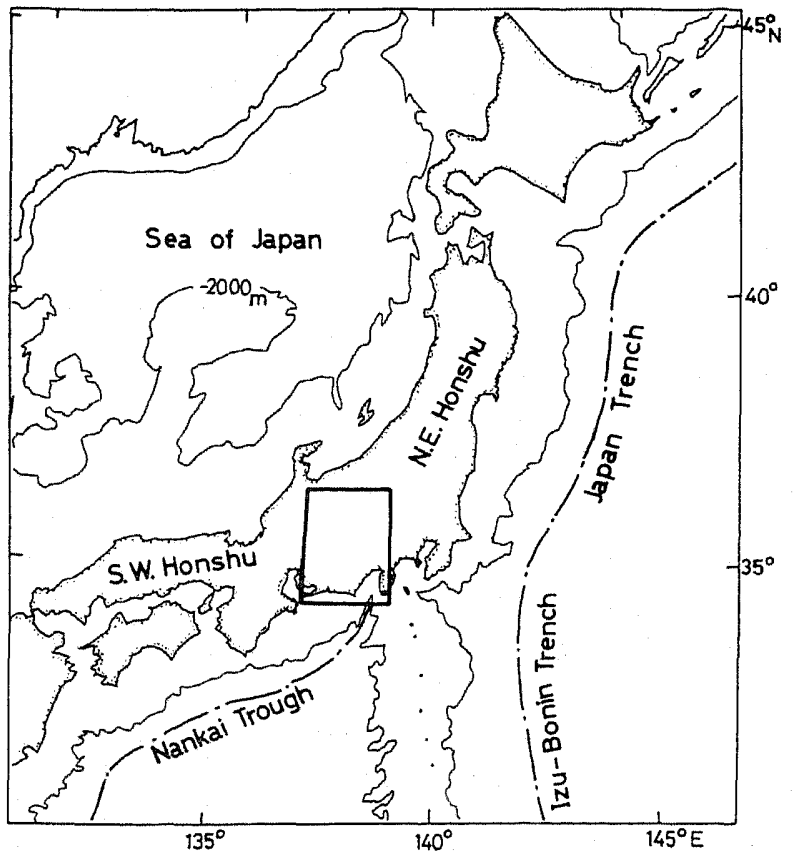

Fig. 1. Map showing the area of survey. 
(OKADA and ANDO, 1979).

Figure 2 shows a geological map simplified from HrRokawa (1978). The Central Ranges have uplifted largely through the late Quaternary. The rate of uplifting is $1-3 \mathrm{~mm} /$ year in the Hida mountains. This is highest rate ever known geologically in Japan (Research Group FOR QUATERNARY TECTONIC MAP, 1973). Even a higher rate- $4 \mathrm{~mm} /$ year - has been reported from geodetic measurements made near the southern periphery of the Akaishi mountain range (DAMBARA, 1971). The uplift in this region has sometimes been discussed in conjunction with the subduction of the Philippine sea plate along the Nankai trough (ANDO, 1975). To the west of the Central Ranges many active faults of predominantly strike sliptype develop, among which the Atera fault (AF), $60 \mathrm{~km}$ long and trending in the NW-SE direction, is one of the most active faults in Japan (RESEARCH GROUP FOR ACTIVE FAULTS, 1980). The Central Ranges are bounded by this fault to the

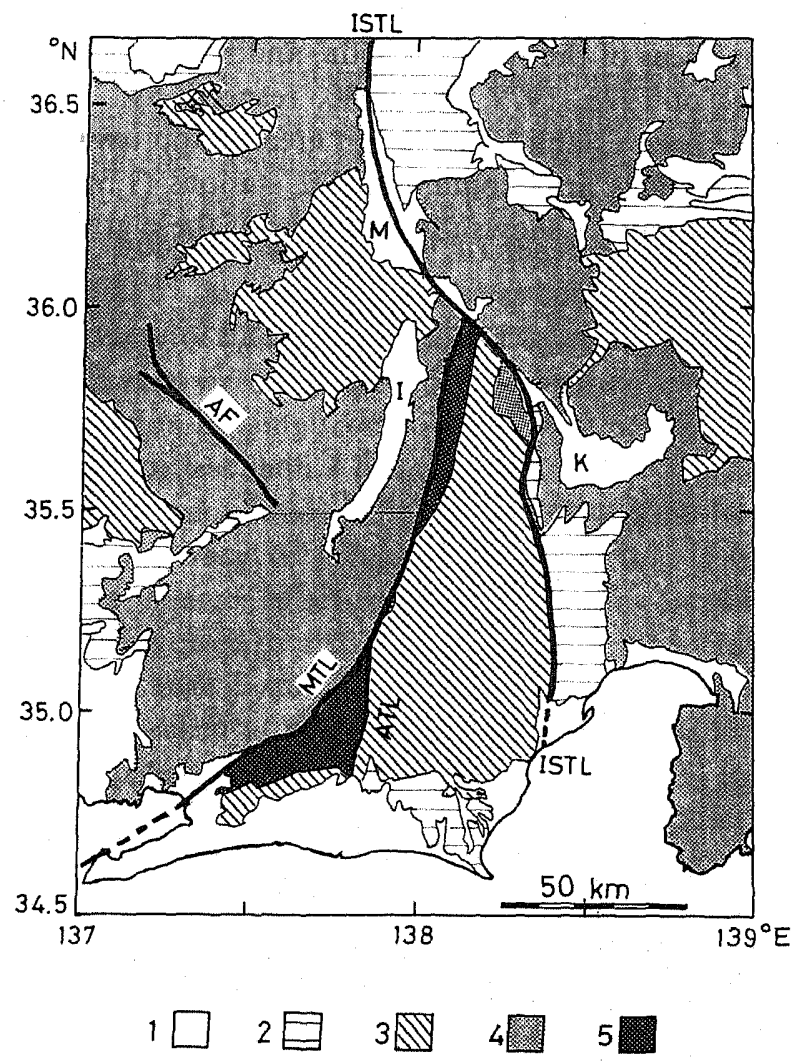

Fig. 2. Simplified geological map of the Central Ranges area after Hrrokawa (1978). 1, Quaternary sediment; 2, Tertiary sediment; 3, basal sediment; 4, igneous rocks and/or metamorphic rocks; 5, metamorphic rocks with ultrabasic rocks (Sanbagawa metamorphic belt); M, Matsumoto basin; K, Kofu basin; I, Ina basin; AF, Atera Fault; MTL, Median Tectonic Line; ISTL, Itoigawa-Shizuoka Tectonic Line; ATL, Akaishi Tectonic Line. 
southwest, where we made a particularly detailed survey. A similar detailed survey has also been carried out along the Median Tectonic Line (MTL) which delineates the western foot of the Akaishi mountain range as a deep and straight fault-line valley (Huzita, 1980). The Central Ranges are bounded by the ItoigawaShizuoka Tectonic Line (ISTL) to the east, which is a huge fault at the western periphery of the Fossa Magna geologically dividing Honshu Island into Northeast Honshu and Southwest Honshu. The Fossa Magna has a complex geologic history but is presently a strongly folded zone forming the Matsumoto sedimentary basin northward and southward, the Fuji River Valley, a thick sedimentary trough (HuzITA, 1980).

\section{Measurements}

Gravity survey was carried out by means of a LaCoste \& Romberg gravimeter, G-484, in the areas of the Akaishi, Hida, and Kiso mountain ranges mainly during the summers of 1978,1979 , and 1980, respectively. In total 2,214 new gravity stations have been established over an area of approximately $2^{\circ} \times 2^{\circ}(137$ $\left.139^{\circ} \mathrm{E}, 34.5-36.5^{\circ} \mathrm{N}\right)$. This area contains 3,722 stations $(1,360$ by GSI and 148 by GSJ) at present. Figure 3 shows the locations of these gravity stations. Few

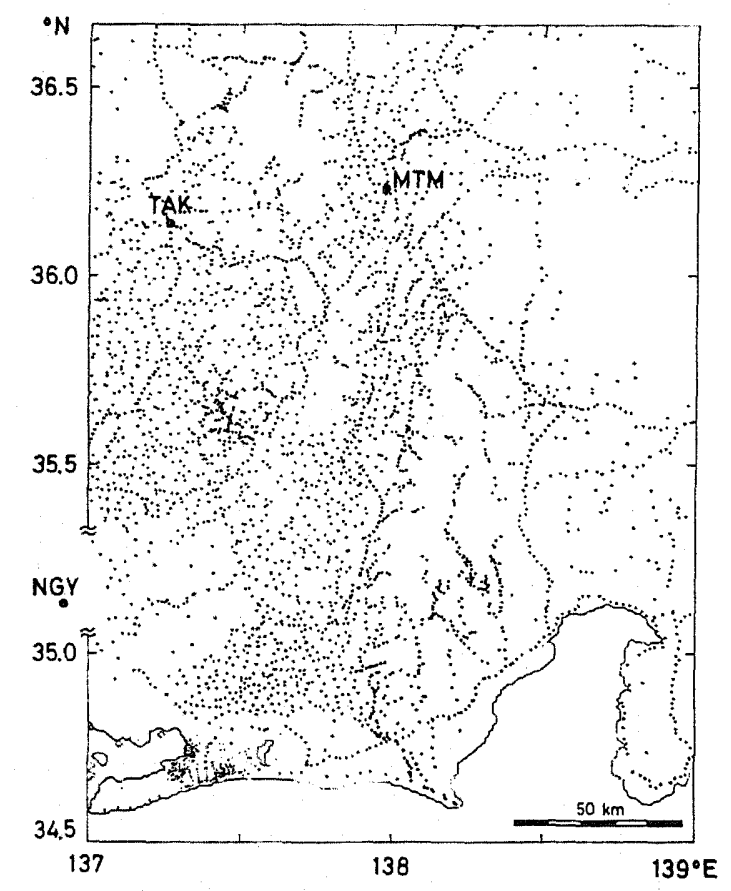

Fig. 3. Locations of gravity stations including the base station (NGY, Nagoya) and the two sub-base stations (TAK, Takayama; MTM, Matsumoto). 
new measurements have been made east of $138.5^{\circ} \mathrm{E}$. About $80 \%$ of the measurements were made at spot heights on $1 / 25,000$ scale topographic maps of the GSI and most of the remaining ones at the bench marks and the triangulation stations installed by the GSI or other public and private institutions. About 130 measurements were made at points where the elevation heights were known only from GSI topographic map contours with a $10 \mathrm{~m}$ interval. The geographical latitude and longitude of each station were read on a map with a precision of $1^{\prime \prime}(\sim 30 \mathrm{~m})$ by an $\mathrm{X}-\mathrm{Y}$ reader.

For the base station of the measurements we used the first order gravity station at Nagoya University $\left(\phi=35^{\circ} 09.1^{\prime} \mathrm{N}, \lambda=136^{\circ} 58.3^{\prime} \mathrm{E}, h=45 \mathrm{~m}\right.$, and $g_{\mathrm{s}}=979,732.63$ mgal) in the Japan Gravity Standardization Net 1975 (GSI, 1976). Since the Hida mountain range area is remote from this base station, we set up two sub-base stations in this area, each of which was linked directly to the base station. The positions of these three stations are shown in Fig. 3. We adopted a closed loop method: the first and the final measurements made during a day were made at the same station. If the two measurements agreed within a certain allowance, the loop was said to be closed. A closed loop was successively linked to the preceding one, the first and the final loops being linked to the base (sub-base) stations. A correction factor of 1.000768 was applied to the scale constants of the G-484 (NAKAGAWA et al., 1981). A measured value was corrected for the tidal variations and the drift rate. Gravity tide was calculated by using the program written by NaKaI (1979). Drift rate was estimated by the method described below, which shows a seasonal variation ranging from -18 to $+30 \mu \mathrm{gal} /$ day. The period of a survey is mostly 1 day, or less than 9 days at most.

The nominal instrumental accuracy of the G-484 is $10 \mu \mathrm{gal}$ (Instruction Manual, 1978). The accuracy of measurement, however, is limited by various conditions. The major sources of error include (a) wrong location of a given spot height, (b) uncertainty involved in drift rate estimation and (c) instrumental "tare" which causes a sudden change in spring length. Since the error due to (a) is serious, it was most carefully checked at every measurement. Moreover we did not use a spot height on a steep slope because a spot height of the GSI is not supported by an actual land mark as accurate as a bench mark or a triangulation station. The relevant error is therefore largely due to the uncertainty of a spot height of 1 to $5 \mathrm{~m}$ dependent upon the conditions of exposure to the sky (personal communication from the GSI, 1981). This uncertainty corresponds to an error of 0.2 to 1.0 mgal in the Bouguer anomaly. The uncertainty associated with (b) is schematically illustrated in Fig. 4. In general the apparent drift rate during a survey, rate 1, is different in a systematic way from that at the base station, rate 2. Rate 1 was determined from the measurements at the base station just before and after a field survey. When a field survey consisted of several loops each of which was closed at a sub-base station, rate 1 was determined for each loop from the measurements made at the sub-base station. We used rate 1 for the calculation of individual gravity values. Rate 2 was determined from a least-squares straight line fit to the 


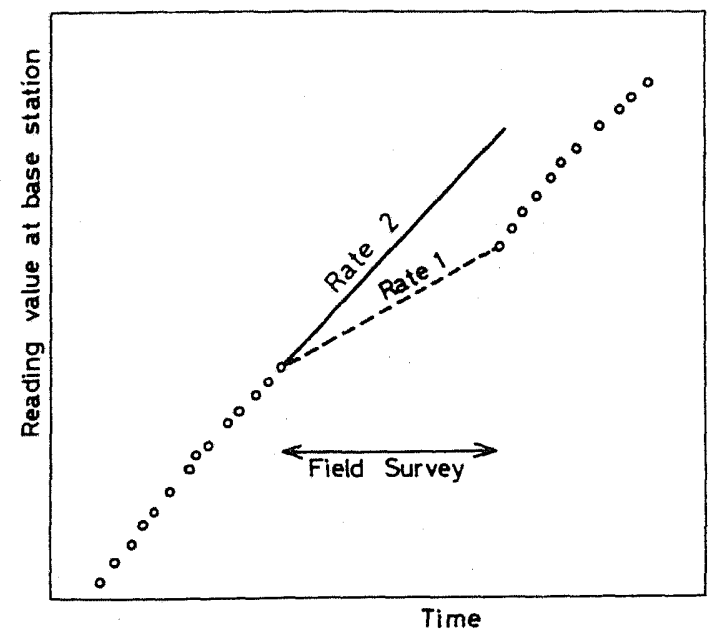

Fig. 4. Schematic illustration for the two methods of drift rate determination. Rate 1 is used for obtaining individual gravity values. If a field survey consists of several loops, each of which is closed at a sub-base station, drift rates are determined for each loop from the measurements made at the sub-base station. Rate 2 is used for calculating the closure errors at the base and the sub-base stations.

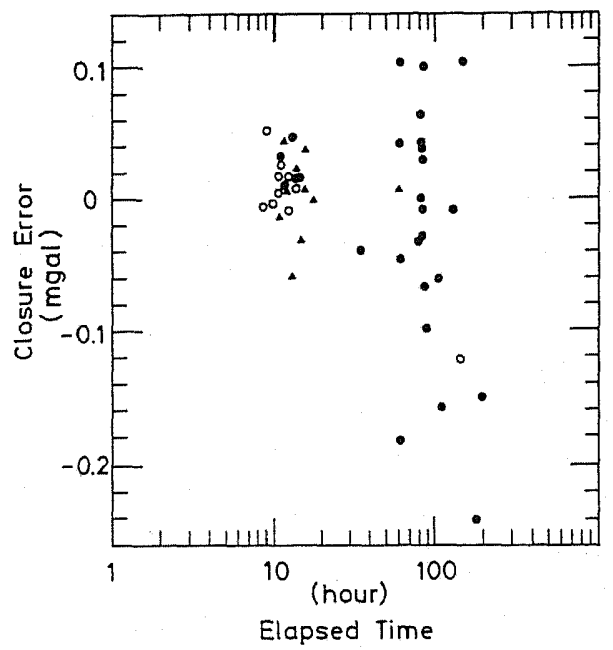

Fig. 5. Observed closure errors against time intervals involved. Solid circles, open circles, and triangles represent closure errors at Nagoya (base station), Takayama (sub-base station), and Matsumoto (sub-base station), respectively.

measurements made at the base station repeated before a field survey. We used this drift rate to calculate closure errors at the base and the sub-base stations. Figure 5 plots the computed closure errors against the relevant time intervals. Most of the closure errors are less than $0.05 \mathrm{mgal}$ for time intervals from several 
to several tens of hours and are less than $0.1 \mathrm{mgal}$ for longer intervals. The errors exceeding 0.1 mgal may be regarded as those due to "tares." The maximum closure errors in 1978,1979 , and 1980 were $0.15,0.24$, and 0.18 mgals respectively, which give some idea of the possible error arising from (c). A closure error as large as $0.24 \mathrm{mgal}$ occurred in a loop containing the highest station $(3,047 \mathrm{~m}$; No. 994 in Appendix), which we climbed carrying a gravimeter on shoulder. In Fig. 5 it is seen that the mean of the closure errors for relatively short time intervals is somewhat different from that for longer time intervals. This difference is interpreted as being due to a systematic difference between the apparent drift rate during a field survey and that before the survey (Fig. 4). The difference is of the order of $0.05 \mathrm{mgal}$ which indicates the uncertainty associated with (b). All the above discussions suggest that the measured gravity values are accurate to $\pm 0.2 \mathrm{mgal}$.

Since the GSI data distribute over the whole area of our survey, it is essential to connect them to our data. We selected 63 GSI stations from various regions and reoccupied them. The difference between the two sets of data was $0.007 \pm$ $0.191 \mathrm{mgal}$. The scatter is relatively large. We, however, note that some of the bench marks might have been moved by several to a few tens of meters after the GSI measurements owing to recent road system development. The standard deviation mentioned above, therefore, would give an upper bound for the real scatter. In any case it is true that we found little systematic difference between the GSI and our data. About 150 GSJ data were available from the southwestern portion of our survey area (CHUJO and SUDA, 1972). They were connected to the GSI data at some overlapping stations (Suda and Chujo, personal communication, 1980).

\section{Bouguer Anomalies}

A Bouguer anomaly value $g$ is evaluated in principle from

$$
g_{\mathrm{B}}=(g-B+T)-(\gamma-\beta h)+\int_{0}^{h} \delta \beta \mathrm{d} z,
$$

where $g$, measured gravity value; $B$, Bouguer correction; $T$, terrain correction; $\gamma$, normal gravity on the geoid; $\beta$, vertical gradient, $\mathrm{d} g / \mathrm{d} z$, of the normal gravity; $h$, station height; and $\delta \beta, \mathrm{d} g / \mathrm{d} z$ anomaly due to mass anomaly below the geoid. In (1) a constant value of $0.3086 \mathrm{mgal} / \mathrm{m}$ was assumed for $\beta$, and $\gamma$ was calculated according to Gravity Formula 1967 (IAG, 1971). The third term related to $\delta \beta$ was neglected in the present study although such a simplification may not be warranted particularly in the area of the Hida mountains where large negative Bouguer anomalies were observed (Fig. 10). The Bouguer anomaly profile across the axis of the Hida mountain range can be approximated by a harmonic wave with a half wavelength of $L=60 \mathrm{~km}$ and a half amplitude of $A=60 \mathrm{mgal}$ (see Fig. 12(a)). The resulting maximum $\mathrm{d} g / \mathrm{d} z$ anomaly is $\delta \beta=-\pi A / L=-0.003$ $\mathrm{mgal} / \mathrm{m}$, which corresponds to free air reduction of $-9 \mathrm{mgal}$ at a station of 
3,000 $\mathrm{m}$ altitude. This value yields a possible upper limit for the error arising from neglecting the $\mathrm{d} g / \mathrm{d} z$ anomaly. For most stations the error would be much smaller.

Usually, the Bouguer correction is made for an infinite slab of thickness $h$. Our survey, however, covered an extensive area of $40,000 \mathrm{~km}^{2}$ with elevation heights ranging from 0 to $3,000 \mathrm{~m}$, for which the earth's sphericity may not be ignored (HAGIWARA, 1975). We made a spherical Bouguer correction over a finite area using the formula presented by HAGIWARA (1975). A spherical terrain correction (TAMADA, 1979) was made in almost the same area as for the Bouguer correction using a modified version of the program written by NozAKI (1981). The program incorporates a terrain data file of the GSI in regular grids with a mesh size of about $230 \times 280 \mathrm{~m}^{2}\left(7.5^{\prime \prime}\right.$ in latitude and $11.25^{\prime \prime}$ in longitude) and changes the computational procedure according to the distance from a measuring point:

(A) In a circular region within a radius of $200 \mathrm{~m}$ from a measuring point the topography is approximated by four to eight cone segments with a common apex at the measuring point as shown in Fig. 6. The basal arc of a cone segment has a common radius of $200 \mathrm{~m}$ at a height level given by the corresponding mesh height. Figure 6 shows the case for the measurement at Mt. Shiomi (No. 994).

(B) For each of the first nine meshes around the measuring point but outside the circular region (A) the topographic mass is approximated by either a right prism or a right prism segment cut by a circular cylinder of a radius $200 \mathrm{~m}$ (see Fig. 6).

(C) Within a distance of $7 \mathrm{~km}$ from the measuring point but outside the rectangular region (B) the topographic mass in each mesh is approximated by a right prism.

(D) Within a distance of $20 \mathrm{~km}$ from the measuring point but outside the region (C) a line mass is assigned to each mesh.

(E) Outside the region (D) a line mass is assigned to every 16 meshes.

The procedures (A) and (B) were checked against a more accurate calculation: the elevation heights in the first nine meshes were read directly from topographic maps at $30 \times 30$ points and further interpolated to produce $300 \times 300$ right prisms for each of which gravity attraction was calculated. We made such a check at three measuring points and one "hypothetical" measuring point. The former includes the summit of Mt. Shiomi (3,047 m; No. 994; see Fig. 6) and two spot heights (Nos. 722 and 740) along the trail of a deep ravine in the Akaishi mountains. The hypothetical measuring point is located at the bottom of an extremely deep ravine immediately below the summit of Mt. Akaishi. We selected these four points because of their steep features nearby. Table 1 shows the result of a comparison between the procedures (A) and (B) and the more accurate calculation described above. The maximum difference occurs at Mt. Shiomi (No. 994), where the procedures (A) and (B) gave a value of $8.4 \mathrm{mgal}$, whereas a more accurate calculation gives a value of $7.2 \mathrm{mgal}$. This result suggests that the error arising from (A) and (B) is less than $1.5 \mathrm{mgal}$ except for some special cases. The pro- 


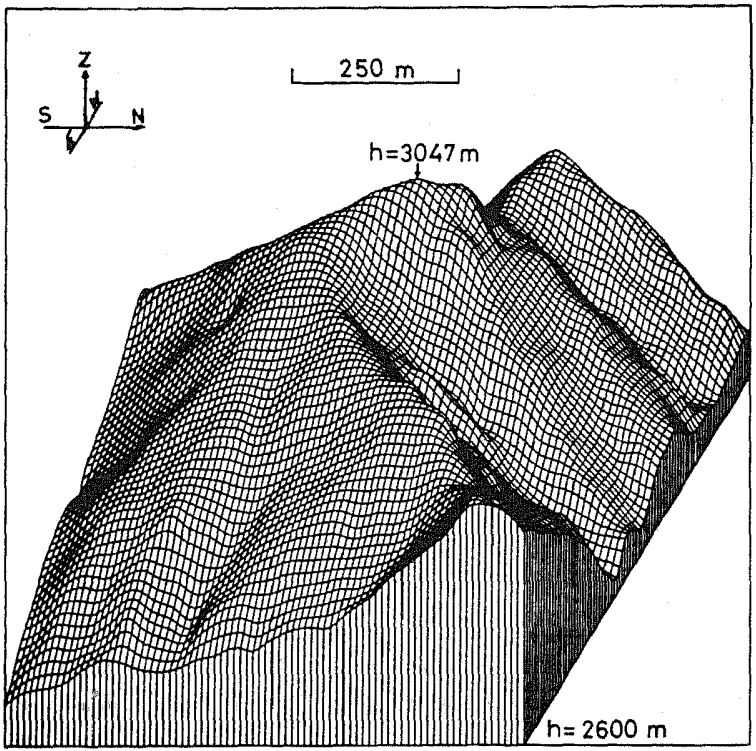

(a)

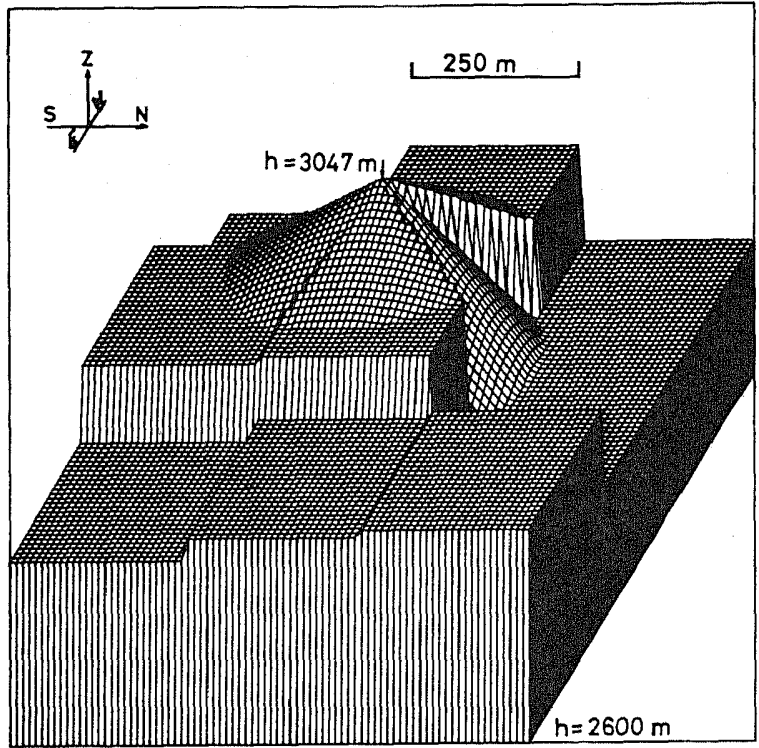

(b)

Fig. 6. Schematic illustration for the correction of topography over the first nine meshes (mesh size $=230 \times 280 \mathrm{~m}^{2}$ ) around a measuring point. (a) Actual topography (Mt. Shiomi, No. 994), (b) approximated topography. 
Table 1. Comparison of terrain correction values between the present method and a more accurate calculation.

\begin{tabular}{cccc} 
& $(\mathrm{A})+(\mathrm{B})$ (mgal) & $\begin{array}{c}\text { More } \\
\text { accurate (mgal) }\end{array}$ & Difference (mgal) \\
\hline No. 722 & 2.2 & 2.4 & -0.2 \\
No. 740 & 2.3 & 1.7 & +0.6 \\
No. 994 & 8.4 & 7.2 & +1.2 \\
No. -* & 7.3 & 7.3 & 0.0 \\
\hline
\end{tabular}

* Hypothetical measuring point.

cedure (C) was checked against a more accurate calculation based on direct reading of elevation heights from topographic maps. Such a check was made at one of the above measuring points (No. 722), suggesting that the error due to (C) is of the order of $0.5 \mathrm{mgal}$. In (D) and (E) the line mass approximation was confirmed to be sufficiently accurate for our purpose.

A question is to what spatial extent the correction should be made in a trade-off between accuracy and computational time. In principle the correction does not converge unless it extends to the whole earth. Our aim is to obtain Bouguer anomaly values that may be biased only constantly for the whole area of survey. We selected several representative stations for each of which Bouguer and terrain corrections $B$ and $T$ were evaluated over a circular region with a radius of $200 \mathrm{~km}$ containing the whole surveying area. We then computed $B$ and $T$ for each of these stations in a distance range $R$ which was successively varied from 5 to $110 \mathrm{~km}$.

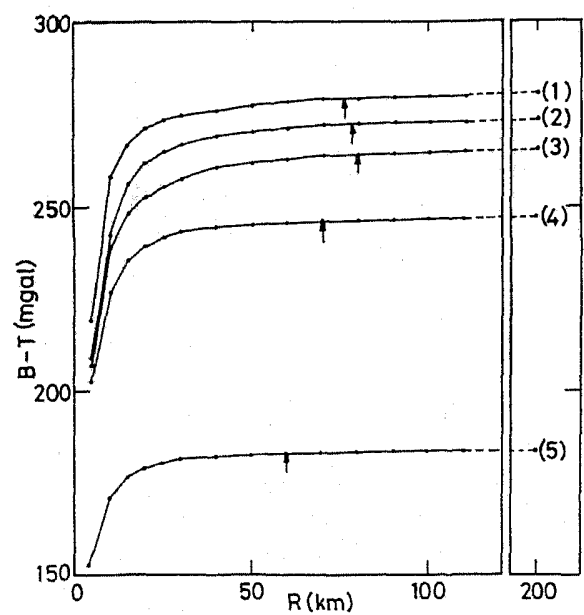

Fig. 7. Correction term $(B-T)$ vs. distance $R$ for several representative stations. The values at $R \sim 200 \mathrm{~km}$ represent those which may be biased only constantly over the area of survey. Arrows indicate the distance at which $(B-T)$ takes a value $1.5 \mathrm{mgal}$ smaller than that at $R \sim 200 \mathrm{~km}$. (1) Mt. Fuji $(3,775.6 \mathrm{~m})$, (2) Mt. Shiomi (3,046.9 m), (3) Mt. Kisokoma (2,956.3 m), (4) Murodo (2,410 m), and (5) Kurobe-Daira (1,828.2 m). 
Figure 7 shows how the correction term $(B-T)$ approaches the one with the ultimate radius of $200 \mathrm{~km}$ as $R$ increases. The difference becomes less than $1.5 \mathrm{mgal}$ at $R=80 \mathrm{~km}$ in all the cases we examined, suggesting that if we take $R=$ $80 \mathrm{~km}$, the corrections can be made with a relative accuracy of $1.5 \mathrm{mgal}$.

Bouguer and terrain corrections must be made with an appropriate value of density as, for example, a density change of $0.2 \mathrm{~g} / \mathrm{cm}^{3}$ causes a gravity difference of about $25 \mathrm{mgal}$ at a station of $3,000 \mathrm{~m}$ altitude. We estimated a true (average) density $\rho_{0}$ for surface features based on our gravity data by a method suggested by FUKAO et al. (1981). The RHS of (1) can be calculated, by neglecting the third term, as a function of assumed density $\rho$ :

$$
\begin{aligned}
g_{\mathrm{B}}(\rho) & =g-B(\rho)+T(\rho)-\gamma+\beta h \\
& =F-H \rho,
\end{aligned}
$$

where

$$
F=g-\gamma+\beta h
$$

is free-air anomaly and

$$
H=B(1)-T(1)
$$

is understood as a topographic factor. The method of Fukao et al. (1981) subdivides the measuring area into meshes of equal size in a virtual attempt to determine a set of mesh-averaged gravity values $\bar{g}_{\mathfrak{B}}$. What then follows is the calculation of an optimum value of $\rho_{0}$ and optimum values of $\bar{g}_{B},\left[\rho_{0}\right]$ and $\left[\bar{g}_{B}\right]$, as functions of mesh size by minimizing in a least-squares sense the differences between $g_{\mathrm{B}}(\rho)$ and $\bar{g}_{\mathrm{B}}$. Figure 8 shows the result of the application of this method which is

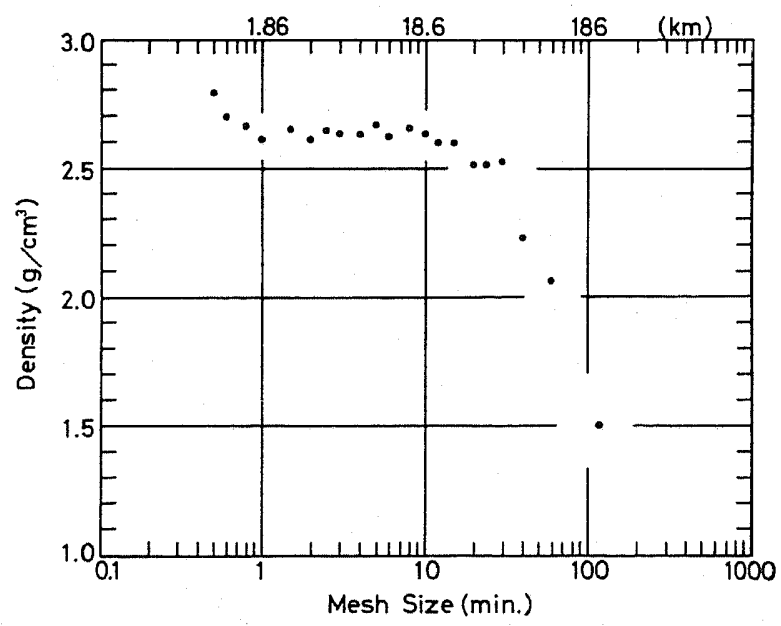

Fig. 8. Plot of an optimum density as a function of mesh size. The mesh size is changed from $0.5^{\prime} \times 0.5^{\prime}$ to $120^{\prime} \times 120^{\prime}$ where $1^{\prime} \times 1^{\prime}=1.53 \times 1.86 \mathrm{~km}^{2}$. The plot indicates a terrain density of about $2.64 \mathrm{~g} / \mathrm{cm}^{3}$. 
slightly different from the earlier result of FUKAO et al. (1981) who included some more inconsistent data. The mesh size was changed from $120^{\prime} \times 120^{\prime}$ (the whole measuring area) to $0.5^{\prime} \times 0.5^{\prime}$. The calculated density $\left[\rho_{0}\right]$ shows an approximately constant value of $2.64 \mathrm{~g} / \mathrm{cm}^{3}$ for mesh sizes from $1^{\prime}$ to $10^{\prime}$. This constancy indicates that the Bouguer anomaly is little correlated with topography in a linear dimension of less than $20 \mathrm{~km}$ and that the Central Ranges area of $2^{\circ} \times 2^{\circ}$ has an average density of $2.64 \mathrm{~g} / \mathrm{cm}^{3}$ for surfaces. For mesh sizes larger than $10^{\prime},\left[\rho_{0}\right]$ decreases rapidly with increasing mesh size although it is still of a finite value of $1.50 \mathrm{~g} / \mathrm{cm}^{3}$ at the largest mesh size. This decrease is diagnostic of a tendency toward isostasy. A still finite $\left[\rho_{0}\right]$ for the whole measuring area, however, suggests that a complete isostasy is not achieved in this limited area.

The effect of lateral variation of density is difficult to evaluate. If, for example, the Matsumoto sedimentary basin at relatively high altitudes of about $600 \mathrm{~m}$ had a density $10 \%$ smaller than the average, the use of the value $2.64 \mathrm{~g} / \mathrm{cm}^{3}$ would have led to an overestimation of the negative anomalies of this basin by an

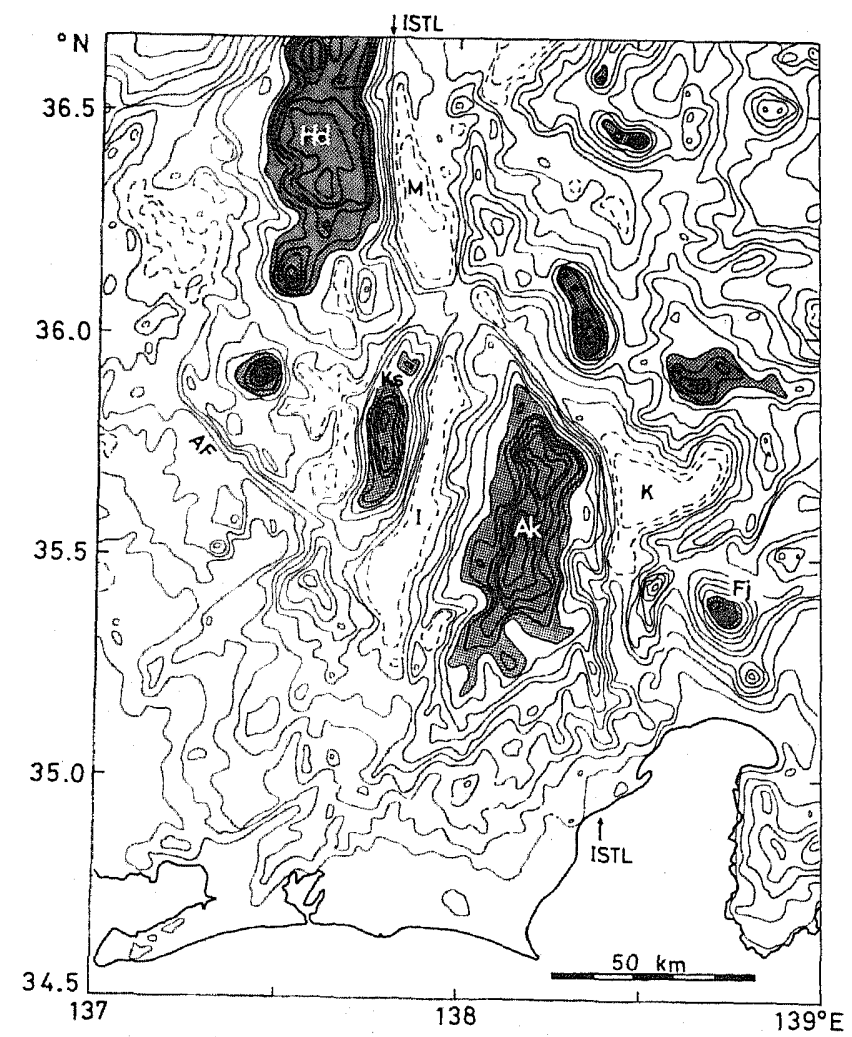

Fig. 9. Summit level map of the Central Ranges area with $200 \mathrm{~m}$ contour (after Research Group for Quaternary Tectonic Map, 1969). A loop with dashes indicates an area of depression. Hd, Hida mountains; Ks, Kiso mountains; Ak, Akaishi mountains; and Fj, Mt. Fuji. See Fig. 2 for other symbols. 
amount of $7 \mathrm{mgal}$. For other sedimentary basins at lower altitudes, the degree of overestimation would be proportionally smaller. Along the above line of discussion we made spherical Bouguer and terrain corrections over a distance range $80 \mathrm{~km}$ using a uniform density of $2.64 \mathrm{~g} / \mathrm{cm}^{3}$. All the gravity values with and without corrections are listed in Appendix.

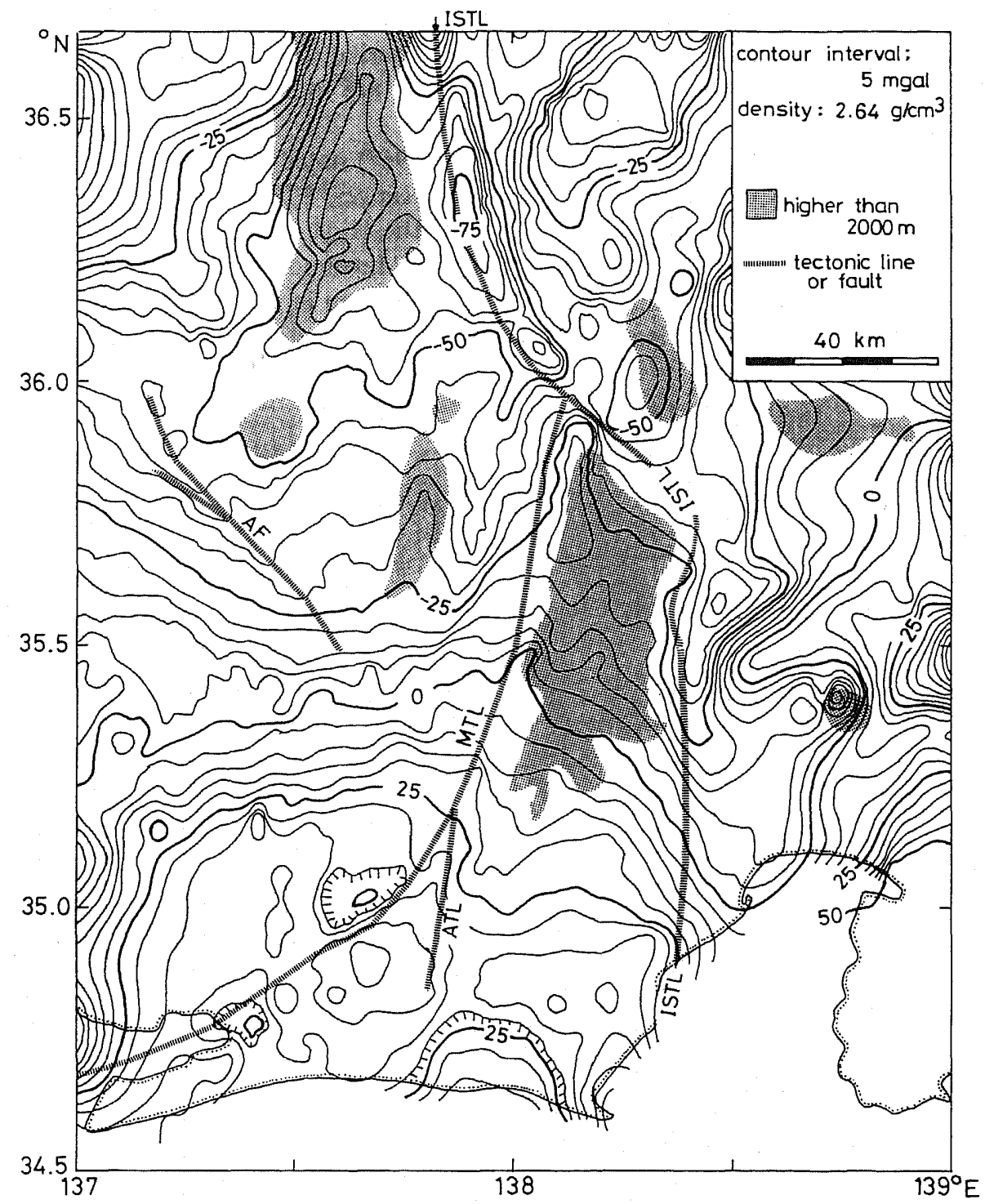

Fig. 10. Bouguer anomaly map with $5 \mathrm{mgal}$ contour. A loop with barbs indicates an area of Bouguer low. Mountainous areas higher than $2,000 \mathrm{~m}$ are indicated by hatching. See Fig. 2 for the explanation of symbols. 


\section{Bouguer Anomaly Map}

Bouguer anomaly values were converted into grid data by a simple averaging procedure. The grid mesh size was $1^{\prime} \times 1^{\prime}$. This data set was subjected to machine contouring using the BRIGGS' (1974) method in which the iteration was repeated 500 times. Figure 10 shows the Bouguer anomaly map with 5 mgal contour so obtained. The summit level map with $200 \mathrm{~m}$ contour is shown in Fig. 9 for comparison. We also constructed $1 \mathrm{mgal}$ contour maps in some restricted regions using the smaller-mesh-size data. We briefly describe several important features of these maps. The areas subject to description are indicated by cross-sections in Fig. 11.

(1) Tsuboi et al. (1955) and Hagrwara (1967) noted a broad zone of large negative anomalies in the area of the Central Ranges. This broad zone is now resolved into two parts, one along the axis of the Hida mountain range and the other along the northern Fossa Magna (the Matsumoto sedimentary basin). Two separated zones of negative anomaly are also seen in Fig. 12(a) which shows the Bouguer anomaly profile across the Hida range for cross-section $\mathrm{AA}^{\prime}$ of Fig.

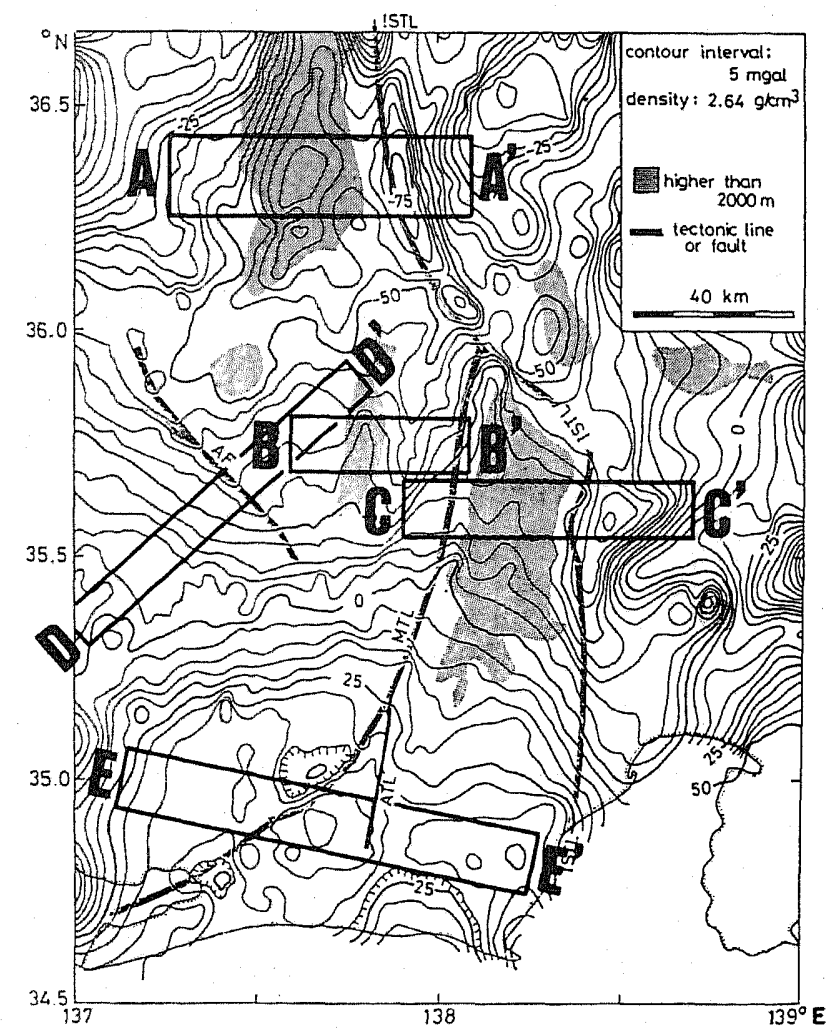

Fig. 11. Bouguer anomaly map with $5 \mathrm{mgal}$ contour (see Fig. 10). The cross-sections indicated appear in Figs. 12-15. 

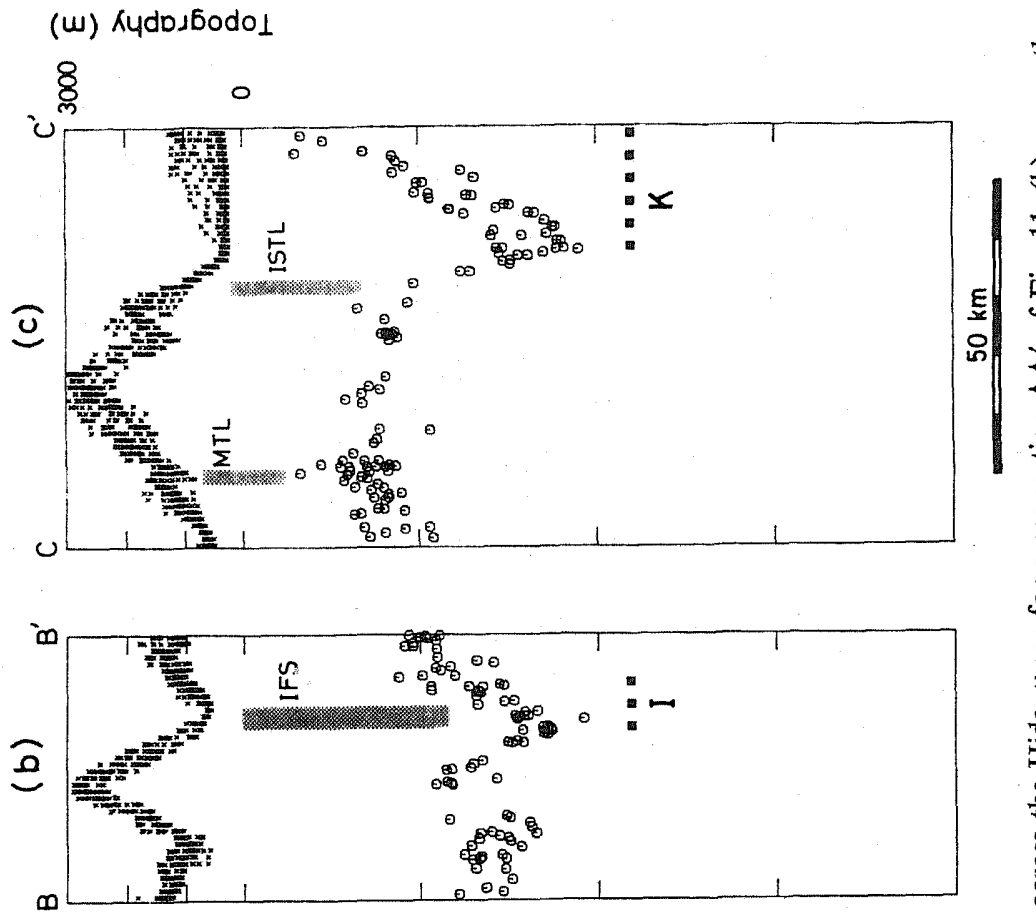

$\cong \stackrel{0}{\varpi}$

岁岁毛

気 量

อ语密

$\exists=$

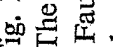

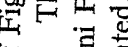

出造密

过范

도원

总

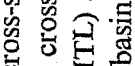

ठ 햘

क

品

可记

ซ

苟焉

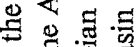

过

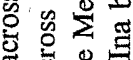

它 击

(ब)

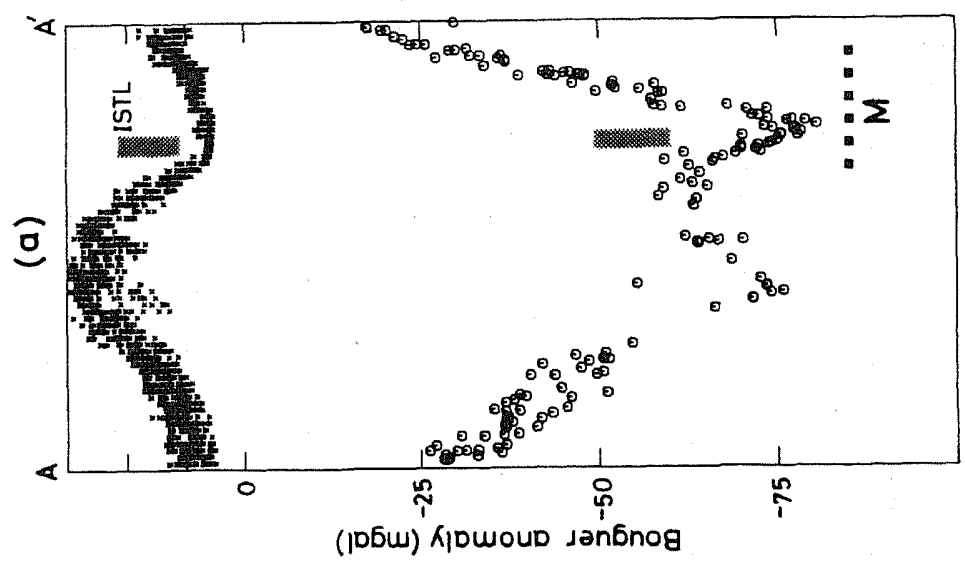

岁完

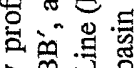

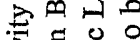

흔홍용

的 巳寸

要

$>$ 要

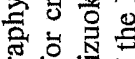

茲 윰

응

合焉

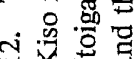

$\approx$ 的

(ןБW) K|DWoud Jan6nog

量 
11. The correction of $\mathrm{d} g / \mathrm{d} z$ anomaly would emphasize more significantly the negative anomaly along the Hida mountain range, while the correction for lateral density variation would reduce the negative anomaly in the Matsumoto basin. The negative anomaly along the Hida mountain range suggests a crustal "root" beneath it, which is, however, not large enough to sustain the overlying topographic mass isostatically (NoZAKI, 1980).

(2) The Central Ranges consist of the Hida, Akaishi, and Kiso mountain ranges. As shown in Fig. 12, the correlation of Bouguer anomaly with topography is strongly negative in the Hida mountains, marginally positive in the Akaishi mountains, and significantly positive in the Kiso mountains. It is intriguing that the mountain system becomes less massive in such an order.

(3) The Hida, Akaishi, and Kiso mountain ranges accompany the Matsumoto basin (the northern Fossa Magna), the Kofu basin (the southern Fossa Magna), and the Ina basin on their eastern feet respectively (see Fig. 2). They are characterized by a strongly negative Bouguer anomaly that can be attributed to thick late Tertiary to Quaternary sediments. The anomaly reaches its maximum near

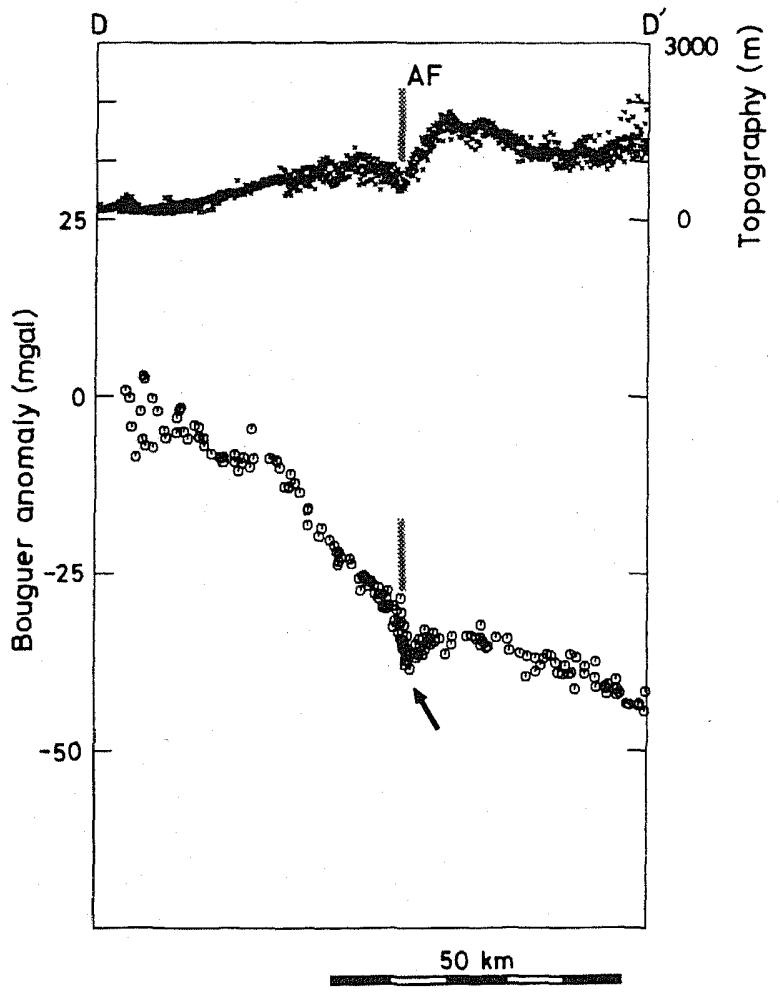

Fig. 13. Topography and gravity profiles across the Atera fault (AF) for cross-section $\mathrm{DD}^{\prime}$ of Fig. 11. Arrow indicates the narrow belt of Bouguer low that can be attributed to the fault gouge materials along the fault. 
the western periphery of each basin as shown in Fig. 12. We note that the Matsumoto and the Kofu basins accompany the ISTL on their western peripheries as. huge thrust faults and that the Ina basin accompanies the Ina valley thrust faults. on its western periphery.

(4) The southwestward extension of the Hida mountain range is morphologically cut sharply by the Atera fault scarp across which there is an abrupt height

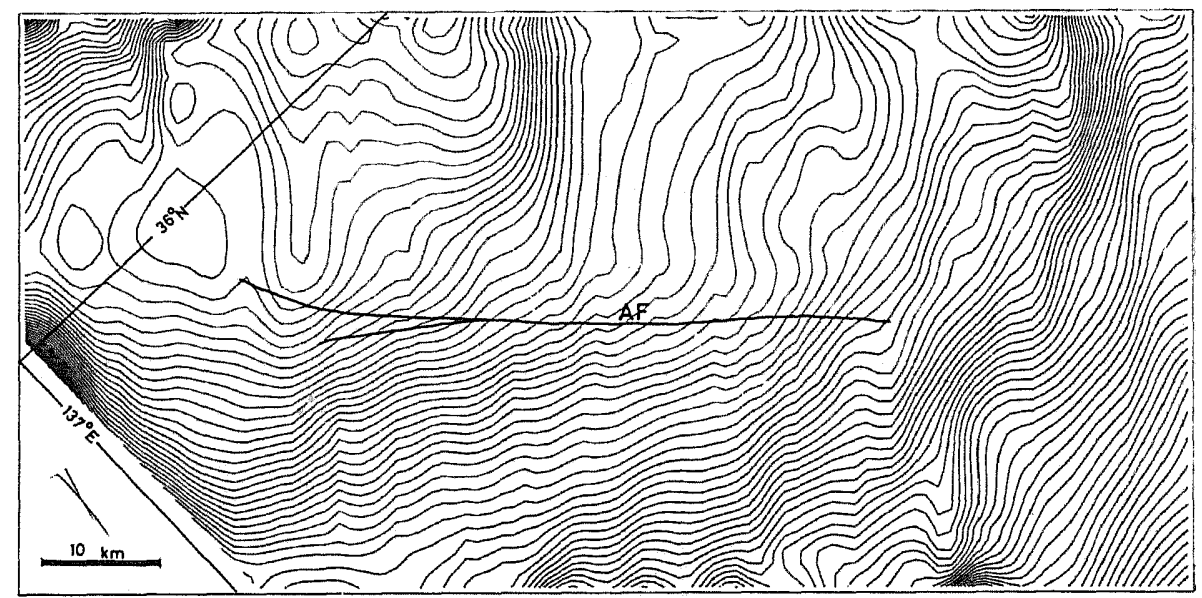

(a)

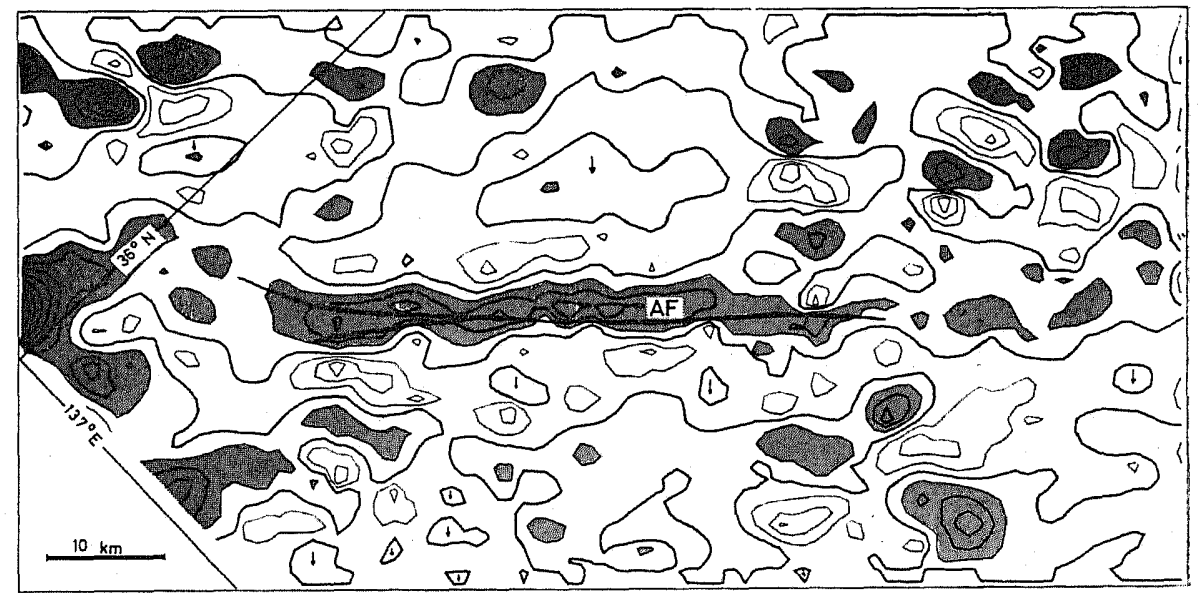

(b)

Fig. 14. Bouguer anomaly maps with 1 mgal contour in the vicinity of the Atera fault (AF): (a) smoothed Bouguer anomaly, (b) residual Bouguer anomaly. Smoothing was done only in the NE-SW direction perpendicular to the strike of the fault. In (b) a thick contour indicates a residual anomaly of $0 \mathrm{mgal}$. An area of residual anomaly of less than $-1 \mathrm{mgal}$ is shown by hatching. Arrow stands for an area of residual anomaly in the range -1 to 0 mgals. 
difference of 700 to $800 \mathrm{~m}$ (see Fig. 9). The negative Bouguer anomaly belt parallel to the Hida range also vanishes southwestward in the vicinity of the Atera fault across which there is a marked difference in regional Bouguer anomaly pattern. Figure 13 shows the topography and gravity profiles across this fault for cross-section $\mathrm{DD}^{\prime}$ of Fig. 11, which clearly indicates that the Atera fault is not only the morphological boundary but also the gravimetric boundary.

(5) The Atera fault is a first-class active fault of the strike-slip type with some dip-slip components, $60 \mathrm{~km}$ long trending in the NW-SE direction, along which a narrow but very clear Bouguer low is observed as indicated by the arrow in Fig. 13, which can be attributed to the fault gouge materials (EZAKA, 1981). To emphasize this narrow belt of Bouguer low, the gravity data are converted into $1 \times 1$ $\mathrm{km}^{2}$ mesh data, which are smoothed over 11 points only in the NE-SW direction perpendicular to the strike of the fault. The differences between the original and smoothed values are then calculated to obtain both the distributions of the smoothed anomaly and the residual anomaly. Figure 14 shows a smoothed map and a residual map with 1 mgal contours. The smoothed map (Fig. 14(a)) clearly

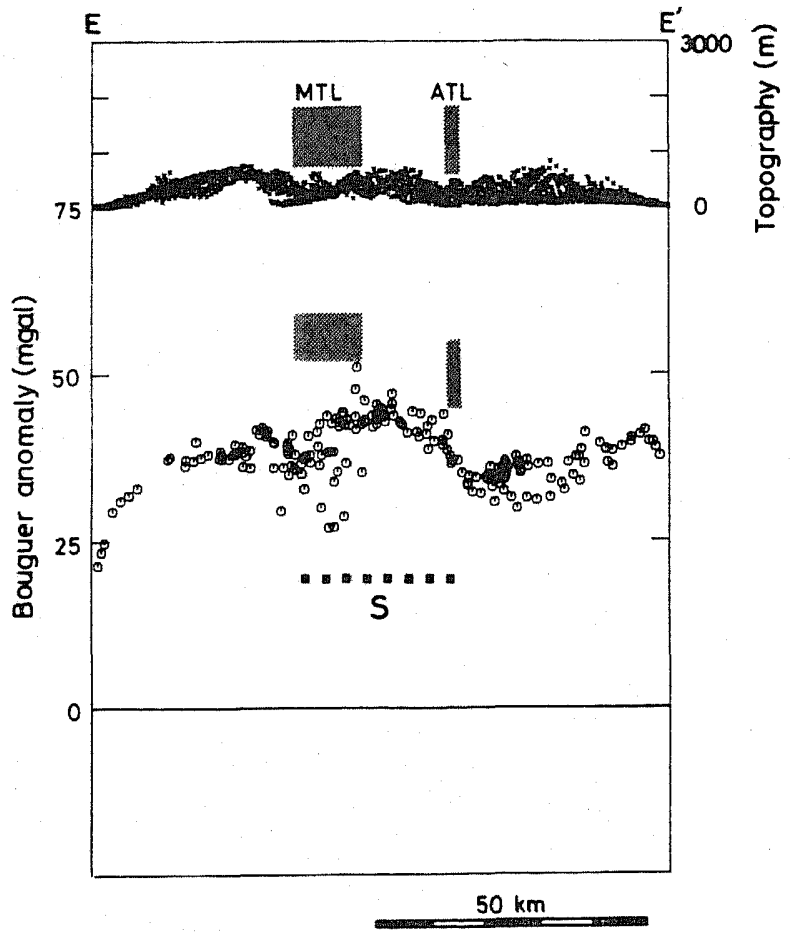

Fig. 15. Topography and gravity profiles across the Median Tectonic Line (MTL) and the Akaishi Tectonic Line (ATL) for cross-section EE' of Fig. 11. The Sanbagawa metamorphic belt (S) is sandwiched by these two tectonic lines. The scatter in Bouguer anomaly near the MLT is due to a Bouguer low in the Shidara basin (see the text). 
indicates a sharp change in regional anomaly pattern across the fault, whereas the residual map (Fig. 14(b)) reveals a narrow belt of Bouguer low along the fault. The axis of this belt does not coincide exactly with the fault trace but shifts toward its upthrown side (mountain side) as can be seen in Figs. 13 and 14(b).

(6) The NS trending MTL accompanies the Sanbagawa metamorphic belt on its eastern side (SUGIMURA and UYEDA, 1973; see also Fig. 2) along which ultrabasic and basic rocks (predominantly serpentinite) are patchily outcropped. A Bouguer high appears to be associated with this metamorphic belt. Such a correspondence is obvious in the southern portion of the MTL where the Sanbagawa belt is relatively wide and is sharply bounded by the Akaishi Tectonic Line (ATL) to the east. Figure 15 shows the topography and gravity profiles across the MTL and the ATL for cross-section EE' of Fig. 11, which indicates an abrupt change in Bouguer anomaly across the ATL. A change across the MTL is much smaller. These features are qualitatively consistent with the results of seismic explosion studies (IKAMI, 1978; INOuE and YAMADA, 1980). As can be seen in Fig. 10, the relative Bouguer high noted above is faced with a relative Bouguer low to the northwest across the MTL. This Bouguer low is well correlated to the Shidara basin of volcanic origin in the Miocene age (KATO, 1962).

\section{Conclusion} follows.

The possible maximum errors involved in our analysis are summarized as

(1) Uncertainty in measured gravity value: $0.2 \mathrm{mgal}$

(2) Error due to uncertainty in elevation height: $1 \mathrm{mgal}$

(3) Uncertainty in terrain correction: $4 \mathrm{mgal}$

Thus the Bouguer anomaly map has an overall accuracy of $5 \mathrm{mgal}$. Other sources of error may arise from the assumption of the absence of $\mathrm{d} g / \mathrm{d} z$ anomaly and lateral density variation.

(4) Possible error due to $\mathrm{d} g / \mathrm{d} z$ anomaly: $10 \mathrm{mgal}$

(5) Possible error due to lateral density variation: $10 \mathrm{mgal}$

Although the maximum errors of the last two sources are large, the errors arising in an actual situation may be much smaller, just as in the case of (3). A large error would have to be taken into account, for example, if one wishes to compare Bouguer anomaly values near coasts to those in high mountains. As for comparison in a narrower region, relative Bouguer anomalies would be regarded in general to be far more accurate.

We pointed out in the last section several outstanding features observed on the Bouguer anomaly map. They will be more fully discussed in a forthcoming paper.

We thank Mr. T. Tsuzuki for his contribution in the early stage of the gravity measurements. We are indebted to all our laboratory colleagues and to the staff of the Microearthquake Observa- 
tories of Nagoya University for their enthusiastic assistance of the survey. Measurements at high altitudes were accomplished in many cases with the generous permission of the district forest offices of the Forestry Agency of Japan, with the cordial assistance of the Hokuriku Electric Power Co., Inc., the Kansai Electric Power Co., Inc., the Tokyo Electric Power Co., Inc. and the Chubu Electric Power Co., Inc. and by the kind arrangements made by various district offices of Nagano, Shizuoka, Gifu, Aichi and Toyama Prefectures, to all of which we express our sincere thanks. Drs. K. Tajima and T. Seto and Drs. J. Chujo and Y. Suda kindly kept us informed on the gravity data respectively obtained by the Geographical Survey Institute and the Geological Survey of Japan. The topographic data file of the GSI was provided by the Japan Map Center. We thank an anonymous reviewer for his valuable comments.

\section{APPENDIX}

The list of gravity values( $G$-value), terrain corrections (T.C.) and Bouguer anomalies (B.A.) is given on pages 222 through 243 . A density of $2.64 \mathrm{~g} / \mathrm{cm}^{3}$ is assumed. Stations are numbered in increasing order of latitude. They are classified into 10 types: blank, spot height; 1 , bench mark; 2, contour; 3 , triangulation station; 4, levelling station (Kansai Electric Power Co., Inc.); 5, levelling station (Tokyo Electric Power Co., Inc.); 6, levelling station (Hokuriku Electric Power Co., Inc.); 7, levelling station (Chubu Electric Power Co., Inc.); 8, levelling station in a tunnel (Kansai Electric Power Co., Inc.); 9, others.

\section{REFERENCES}

ANDo, M., Possibility of a major earthquake in the Tokai district, Japan and its pre-estimated seismotectonic effects, Tectonophysics, 25, 69-85, 1975.

BrigGs, I. C., Machine contouring using minimum curvature, Geophysics, 39, 39-48, 1974.

Chujo, J. and Y. SudA, Gravitational survey of southern Ise Bay and Mikawa Bay, Bull. Geol. Surv. Japan, 23, 573-594, 1972 (in Japanese).

Dambara, T., Synthetic vertical movements in Japan during the recent 70 years, J. Geod. Soc. Japan, 17, 100-108, 1971 (in Japanese).

EZAKA, T., Bouguer anomalies around the Atera fault and along the eastern part of the Median Tectonic Line, Master Thesis, Nagoya University, 1981 (in Japanese).

FuKaO, Y., A. YAMAMOTO, and K. NoZAKr, A method of density determination for gravity correction, J. Phys. Earth, 29, 163-166, 1981.

GeOGRAPHICAL SURVEY INSTITUTE, Gravity survey in Japan, Parts 3 and 4, Gravity survey in Kanto and Chubu districts, Bull. Geogr. Surv. Inst., 9, 155-340, 1964.

GeOGRAPHICAL SURVEY InSTITUTE, Establishment of the Japan Gravity Standardization Net 1975, J. Geod. Soc. Japan, 22, 65-76, 1976.

HaGrWARA, Y., Analyses of gravity values in Japan, Bull. Earthq. Res. Inst., 45, 1091-1228, 1967.

Hagrwara, Y., Conventional and spherical Bouguer correction, J. Geod. Soc. Japan, 21, 16-18, 1975 (in Japanese).

Hirokawa, O. (Chief ed.), Geological Map of Japan, $1: 1,000,000$ (2nd Ed.), Geological Survey of Japan, 1978.

HuzitA, K., Role of the Median Tectonic Line in the Quaternary tectonics of the Japanese islands, Mem. Geol. Soc. Japan, 18, 129-153, 1980.

IkAm, A., Crustal structure in the Shizuoka district, Central Japan as derived from explosion seismic observations, J. Phys. Earth, 26, 299-331, 1978. 
INOUE, H. and I. YAMADA, Crustal structure in the southern Chubu district as derived from explosion seismic observations, Japanese abstract for the 1980 annual meeting of the Seismological Society of Japan, No. 2, p. 154, 1980.

International Association of Geodesy, Geodetic Reference System 1967, Publ. Sóec., 3, Bull. Géod., 116 pp., 1971.

KATo, Y., On the structural development of the Shidara Basin, J. Earth Sci. Nagoya Univ., 10, $51-69,1962$.

Nakagawa, I., H. Tajima, S. Izutuya, Y. Kono, S. Nakai, T. Higashi, R. Shichi, H. Fummoto, K. TAJima, and M. FunaKi, Precise calibration of scale constants of LaCoste \& Romberg gravimeters and international gravimetric connection along the Circum-Pacific zone (the first half of the main investigation), Report on "Precise calibration of scale constants of LaCoste \& Romberg gravimeters and contribution to the reform of the International Gravity Standardization Net 1971 (Main investigation in 1980)," 24 pp., 1981.

NaKaI, S., A subroutine program for computing the tidal forces for the practical use, Proc. Int. Lat. Obs. Mizusawa, 18, 124-135, 1979 (in Japanese).

NozAKr, K., Bouguer anomalies in and around the Akaishi and Hida mountain ranges, Master Thesis, Nagoya University, 1980 (in Japanese).

NozAKI, K., A computer program for spherical terrain correction, J. Geod. Soc. Japan, 27, 23-32, 1981 (in Japanese).

OKADA, A. and M. ANDo, Active faults and earthquakes in Japan, Kagaku, 49, 158-169, 1979 (in Japanese).

ResEARCh Group for Actrve Faults, Active Faults in Japan, 363 pp., The University of Tokyo Press, Tokyo, 1980 (in Japanese).

ReseARCH GROUP FOR QUATERNARY TeCtonIC MAP, The Quaternary Tectonic Map, the National Research Center for Disaster Prevention, Tokyo, 1969.

RESEARCH GROUP FOR QUATERNARY TECTONIC MAP, Explanetory text of the Quaternary tectonic map of Japan, the National Research Center for Disaster Prevention, Tokyo, 1973.

SUGmuRA, A. and S. UYEDA, Island Arcs-Japan and Its Environs, 235 pp., Elsevier, Amsterdam, 1973.

TAMADA, O., Computer program for the reduction of gravity, Tsukumo-chigaku, 14, 48-58, 1979 (in Japanese).

Tokyo Astronomical Observatory (ed.), Rika-nenpyo, Maruzen, Tokyo, 1980 (in Japanese),

Tsuboi, C., A. Jitsukawa, H. Tajima, and A. OKadA, Gravity survey along the lines of precise levels throughout Japan by means of a Worden Gravimeter, Part VI, Chubu District, Bull. Earthq. Res. Inst., Suppl., IV, 199-310, 1955.

\section{Note Added in Proof}

After the completion of this work, the gravity value of the first order gravity station at Nagoya University has been revised $\left(g_{\mathrm{S}}=979,732.54 \mathrm{mgal}\right.$; TOKYO AsTRONOMICAL OBSERVATORY, 1980). The gravity values listed in Appendix are referred to this revised standard. 
(⿻)丨子

䐻

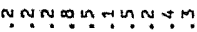
insingising in

$\sin 0$

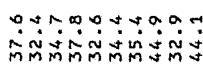

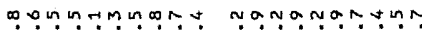

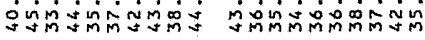

岁鬲

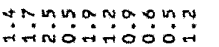

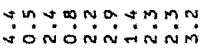

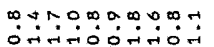

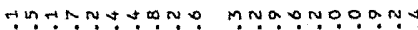

Nôn

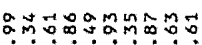

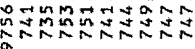

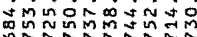

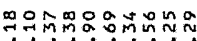

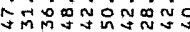

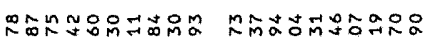
a

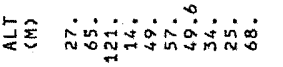

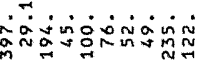

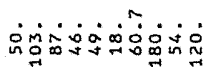

osं

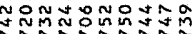

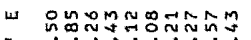

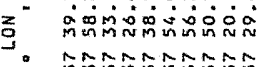

mun

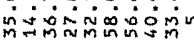

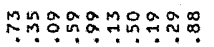

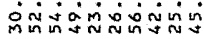

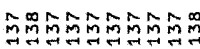

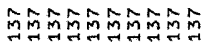

N

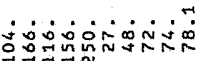

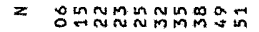

ํำ

in in

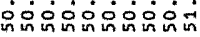

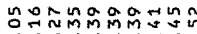

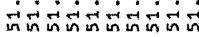

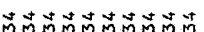

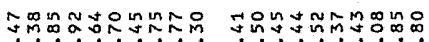
mỡ

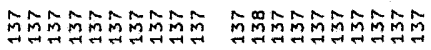

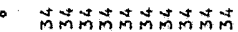

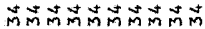

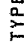

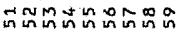

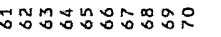

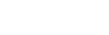

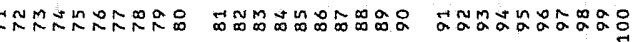

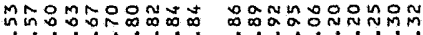

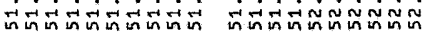

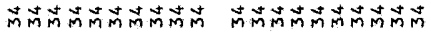

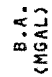

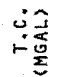

岂表

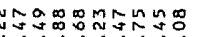

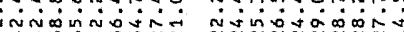
各

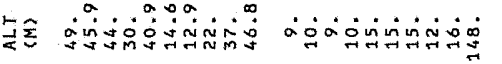

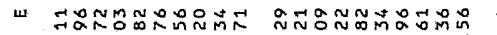

䓃

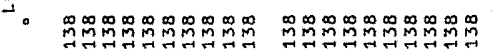

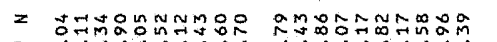

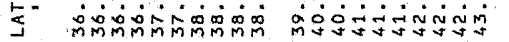

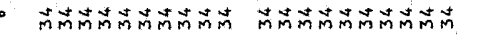

$\frac{4}{2}$

울

\section{H MHA NM}

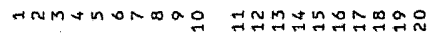

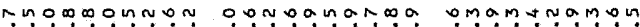
ํำ

YMmmM Ymmmm MmY 00000n0000 0000000000 0000000000

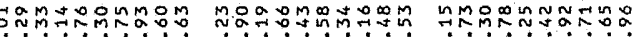

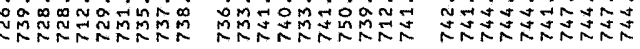

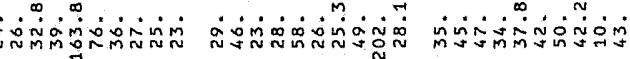

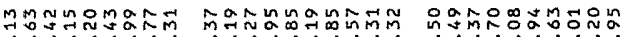
نं

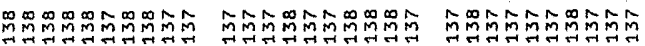

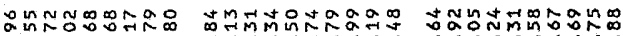

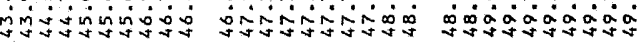

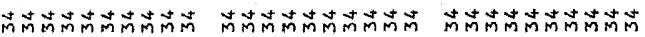

H TN

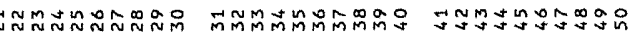




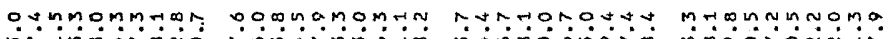

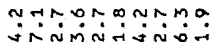

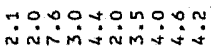

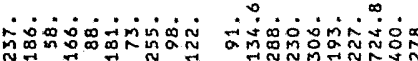

min่⿻i口ing

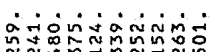

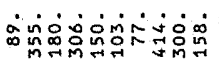

แ popagopơ

ํำㅇำ

s.

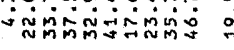

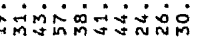

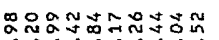

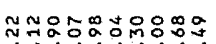

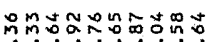

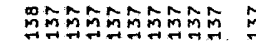

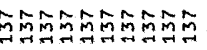

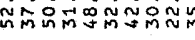

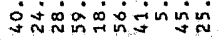
मำ

$z$

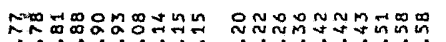

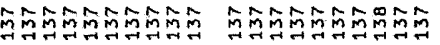

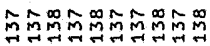

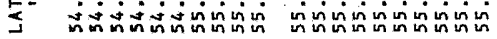

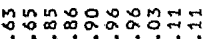

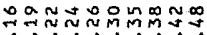

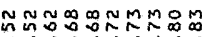

-

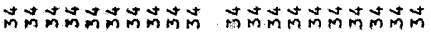

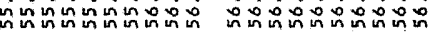

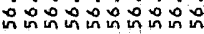

站

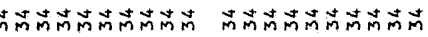

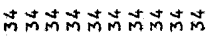

$m$

mN

$\sim$

n

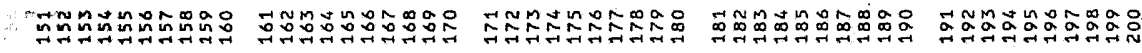

ONONONOMUR mmNONMnOA

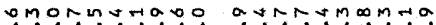

nom in N In NON

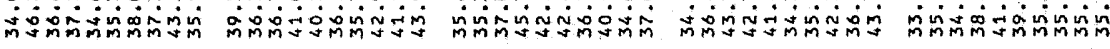

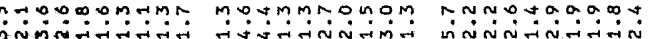

nod

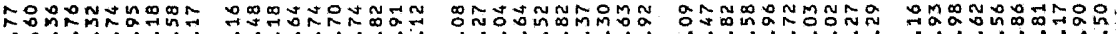

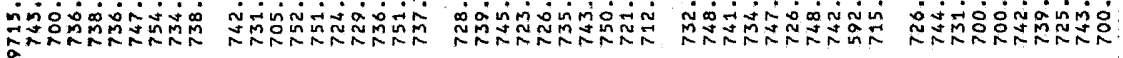
$\underset{a}{a}$

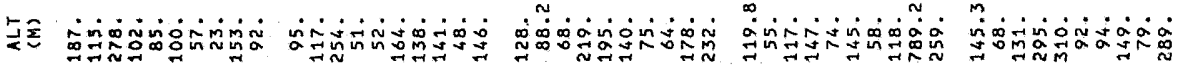

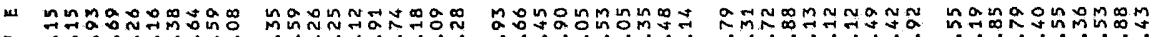

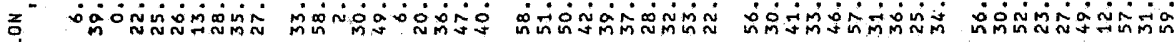

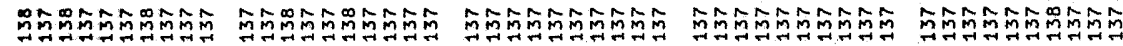

z

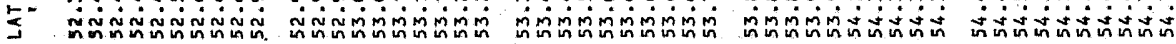

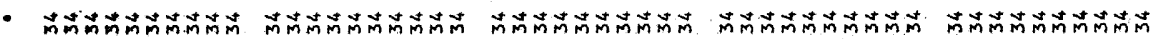

$\stackrel{2}{2}$

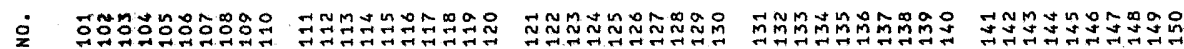




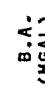

is:

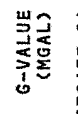

華全

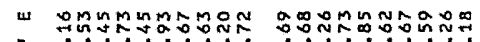

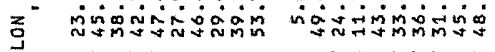

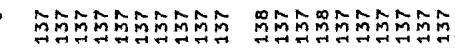

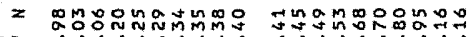

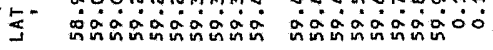

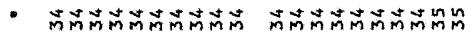

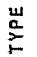

安

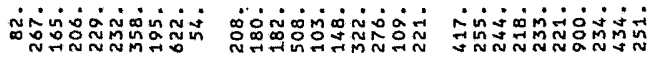

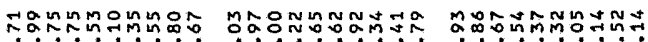

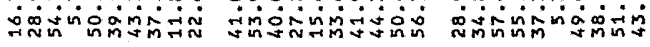

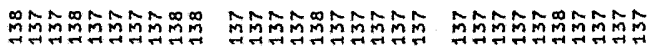

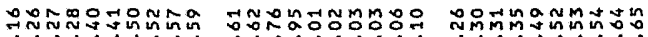

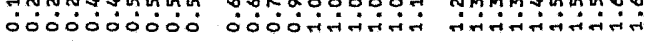

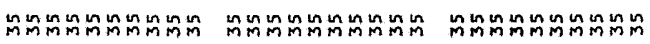

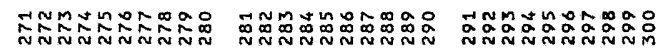

篙

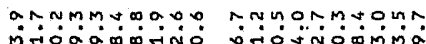
מ.

施

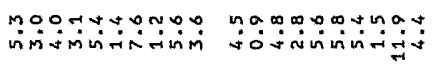

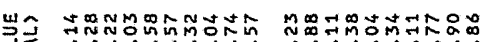

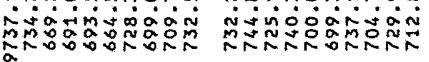
a

w

吾。

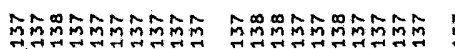

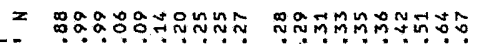

5.

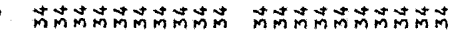

$\frac{21}{2}$

운

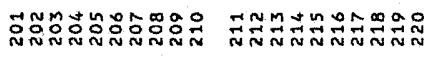

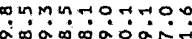
किल

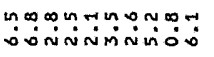

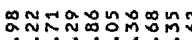

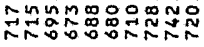

mํํํํำํำำำ

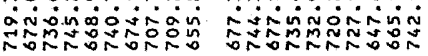

Nูกำ

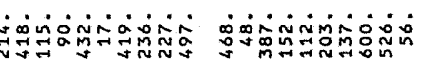

a oñ

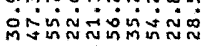

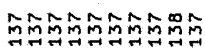

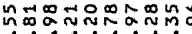

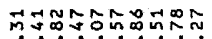

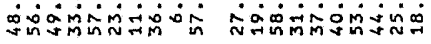

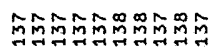

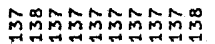

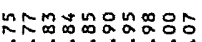

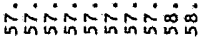

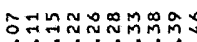

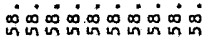

웅ํำ

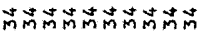

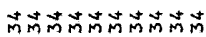

⿻日禸

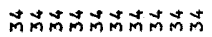

$n$

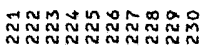

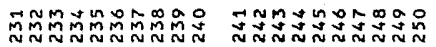



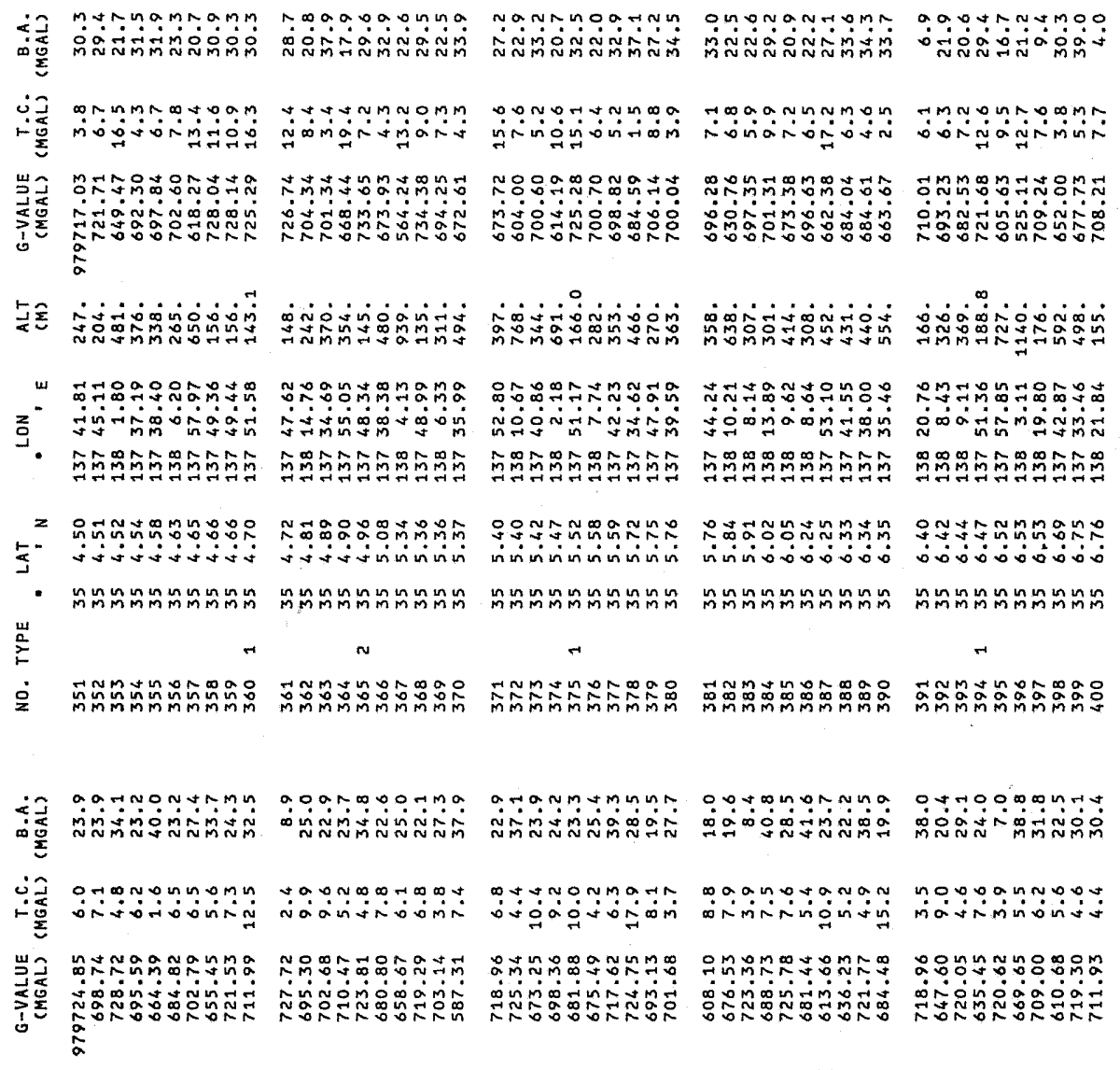

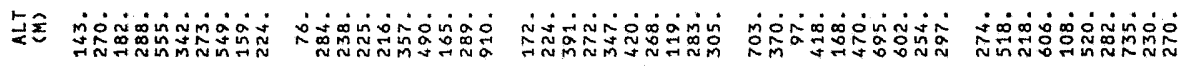

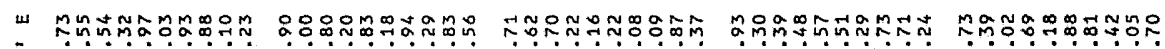

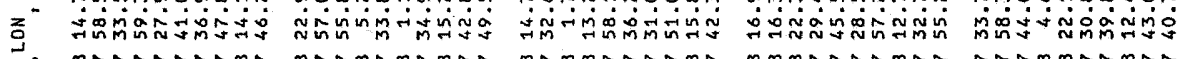

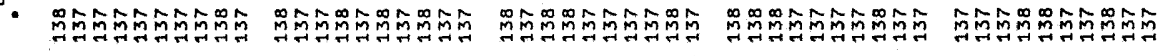

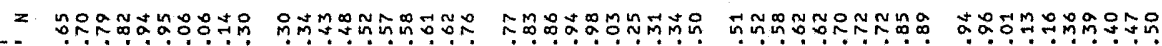

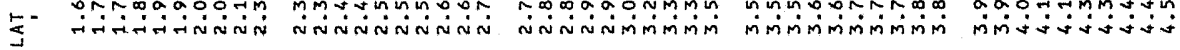

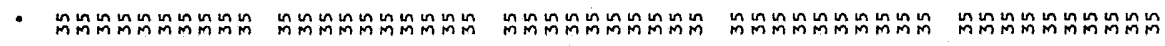

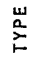

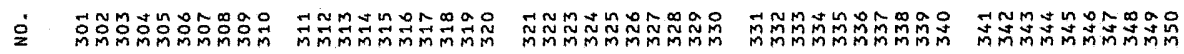


sis

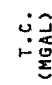

$4 \quad$ in คิ

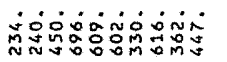

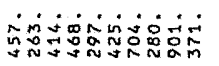

\section{ș.}

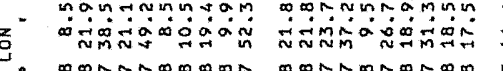
mon

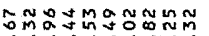

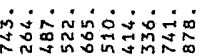

กุด

sto

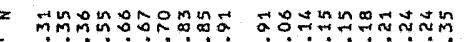

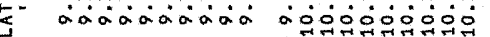

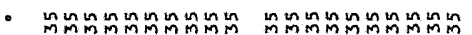

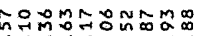

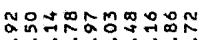

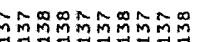

N

क्ष

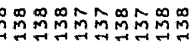

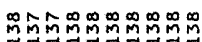

$\stackrel{\substack{0 \\ 2}}{2}$

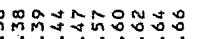

o

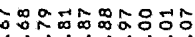

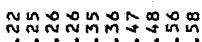

年

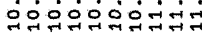

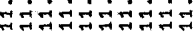

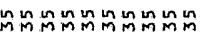

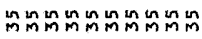

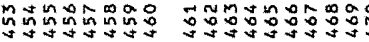

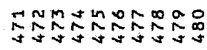

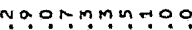

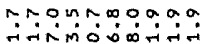

munorrom 000

ayaramono

จำ

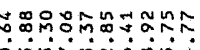

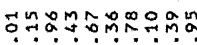

ज्ञ

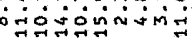

कิ aे

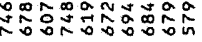

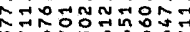

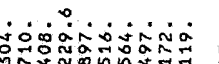

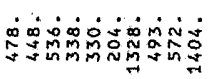

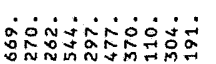

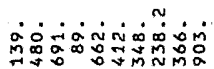

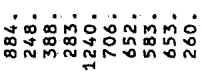

- 웅ำ

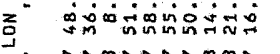

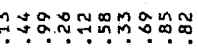

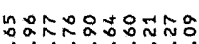

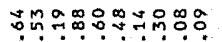

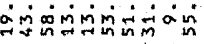

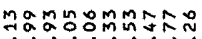

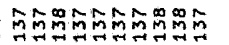

sîn

मिं

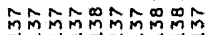

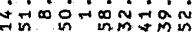

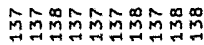

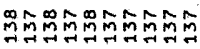

$z$ ก⿻上丨

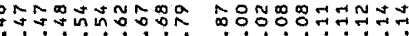

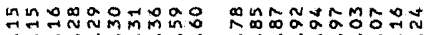

I

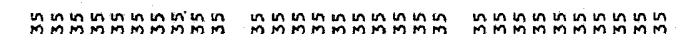

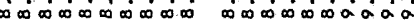

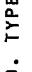

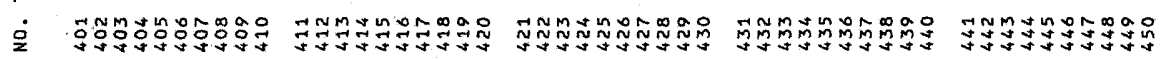




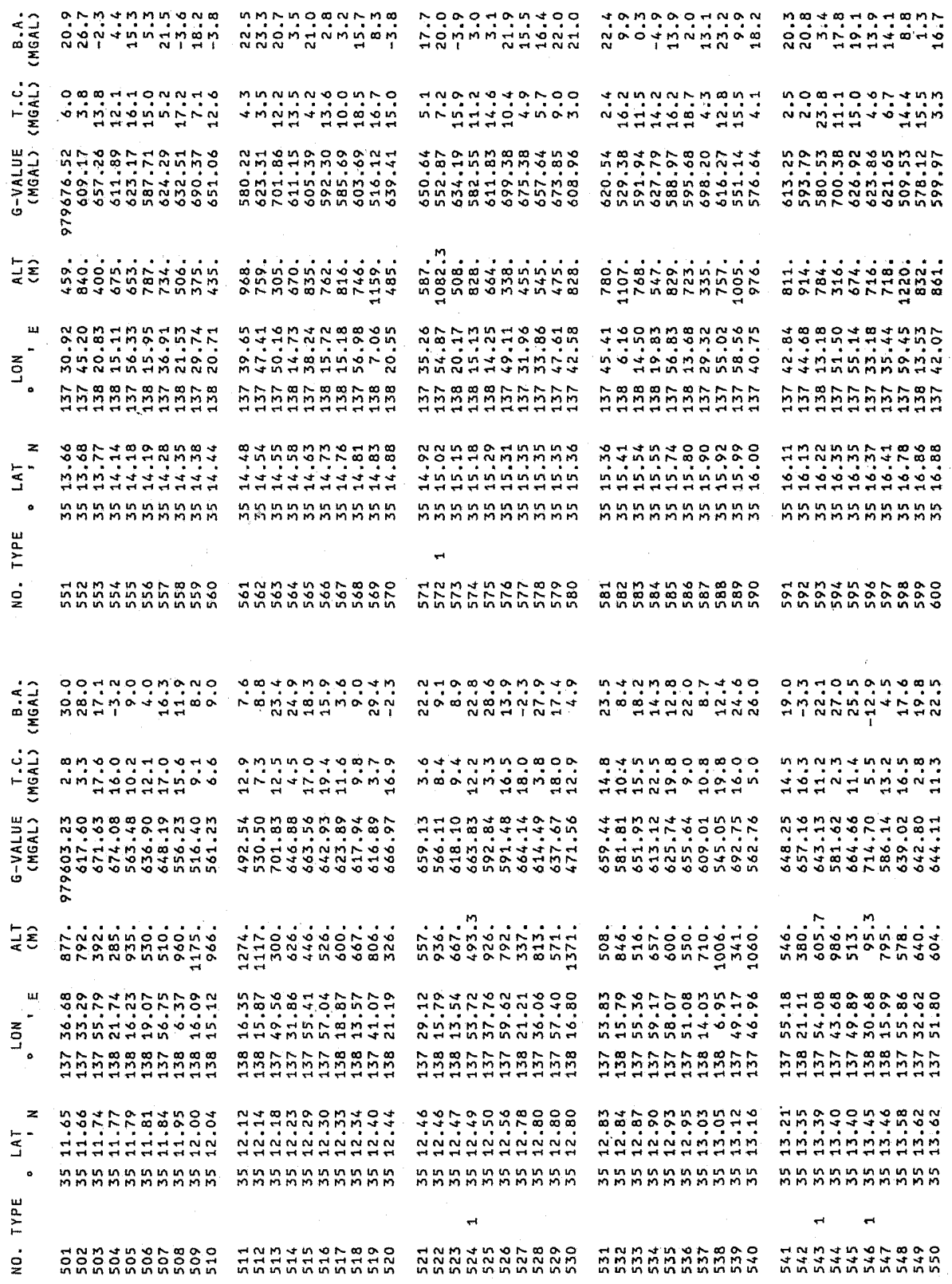




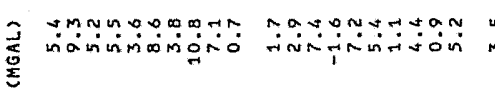

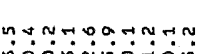

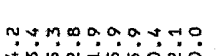

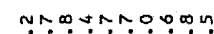

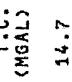

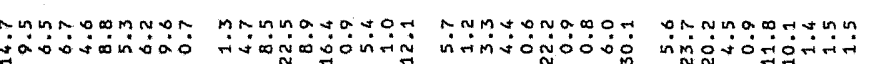

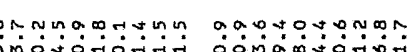

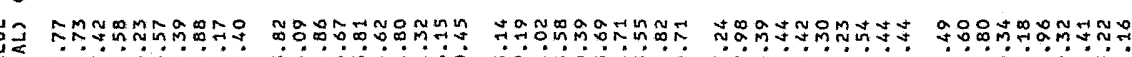

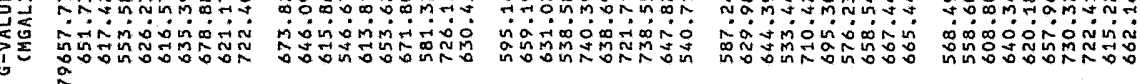
a

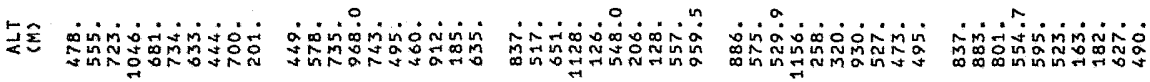

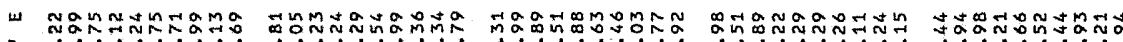

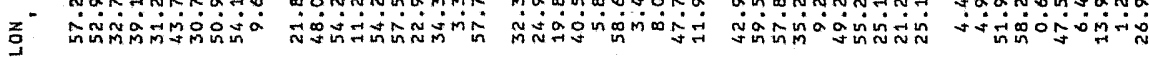

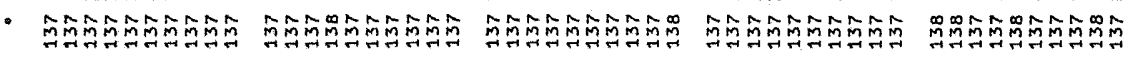

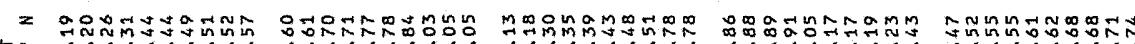

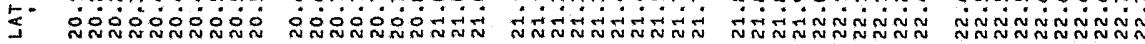

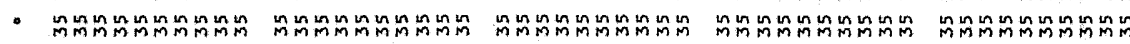

$\stackrel{u}{\overrightarrow{2}}$

n n nNo

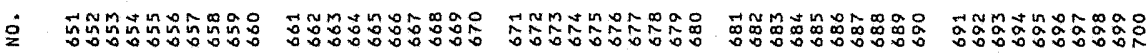

这通

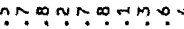

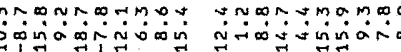

热

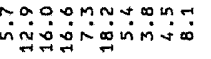

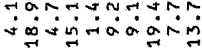

(1)

嵌

maำ

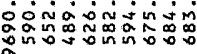
ลั

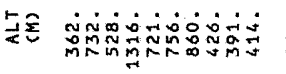

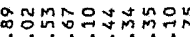

inmiñ

artsing

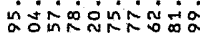

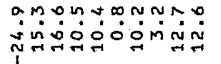

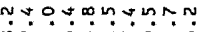

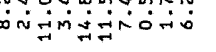

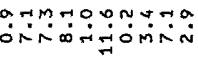

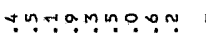

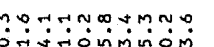

0000000 in 00 to

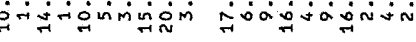

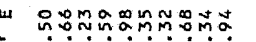

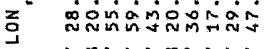

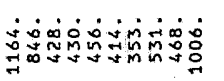

M $\dot{x} \dot{0} \dot{0} \dot{0}$

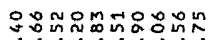

F⿻ino

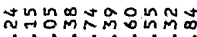

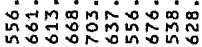

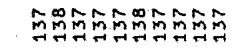

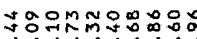

Siminisis inisi

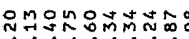

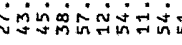

in

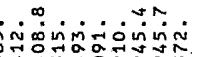

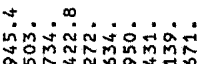

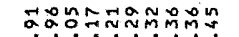

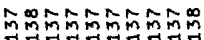

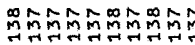

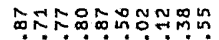

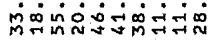

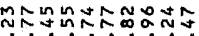

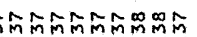

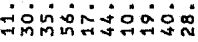

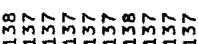

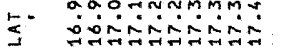

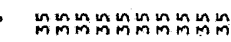

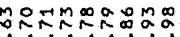

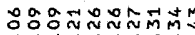

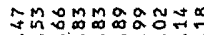

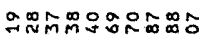

निन्निज्नित्जिजन

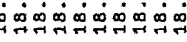

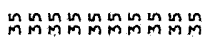

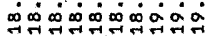

जनेन नें

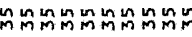

n N. N

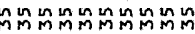

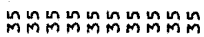

崩

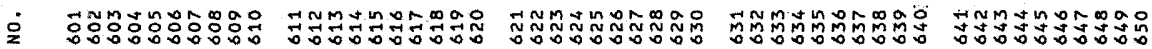




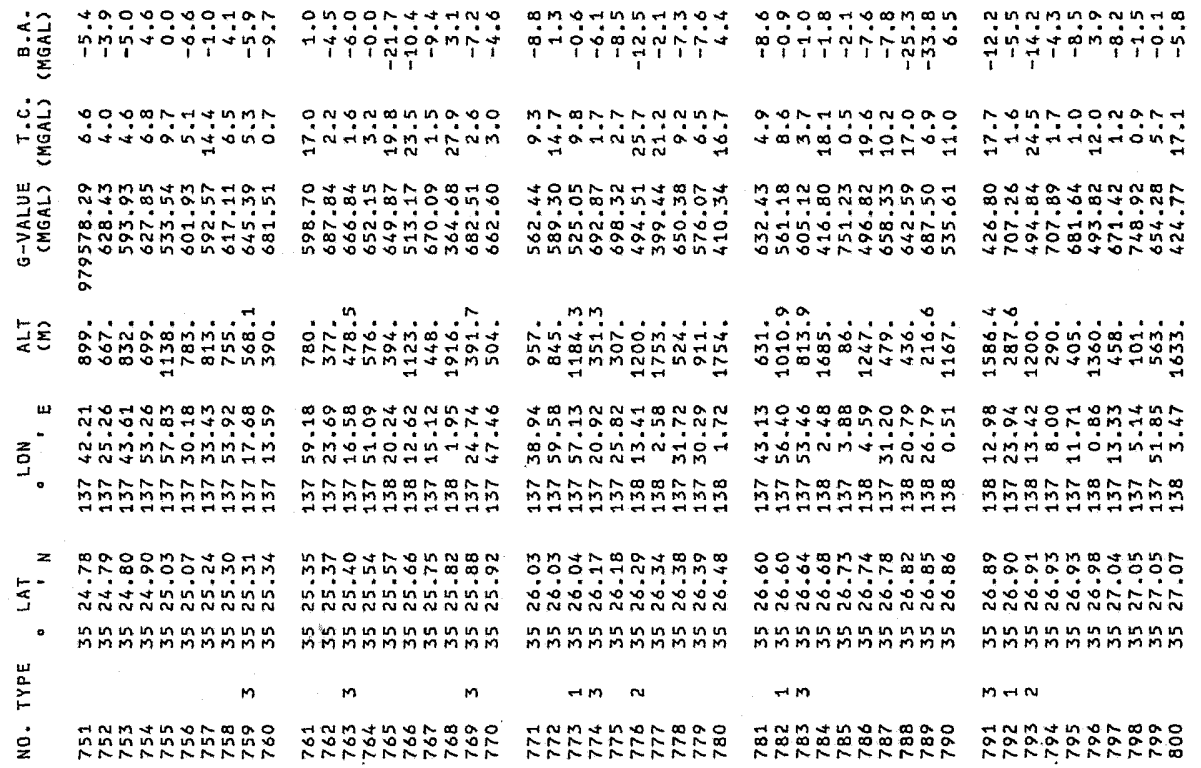

เุ่า

施善

a

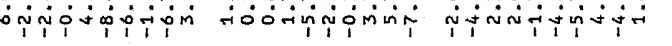

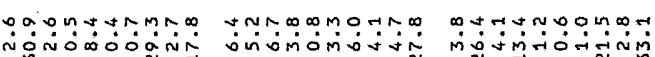

$m$ M

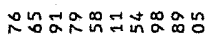

롤

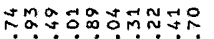

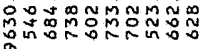

Munuñm-iñ

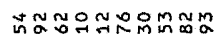

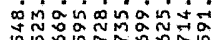
$\frac{a}{a}$

齿

山

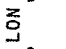

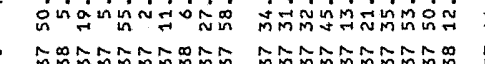

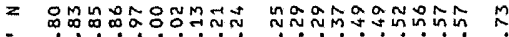

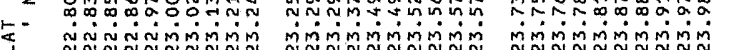

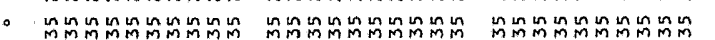

$\stackrel{m}{2}$

$\dot{q}$

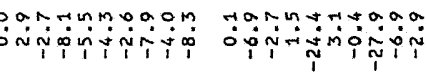

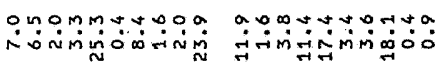

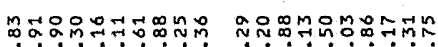

ตำ

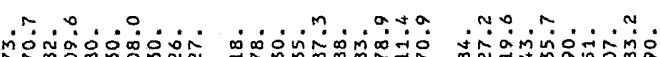

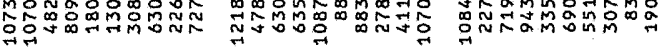

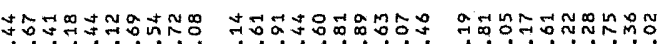

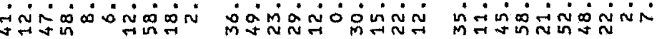

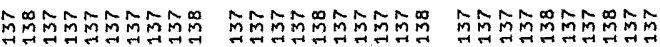

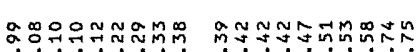

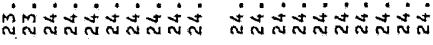

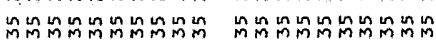

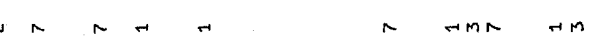

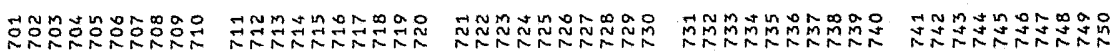


$\rightarrow \ln 0 m m\{00 x$

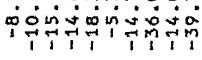

nmpomonos

minmonsunmo

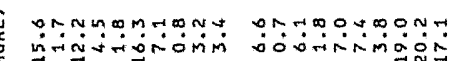

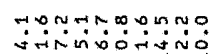

ơntoopran

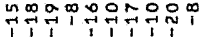

岁

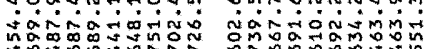

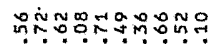
$\overbrace{\substack{a \\ a}}^{a}$

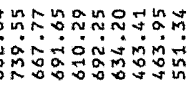

至

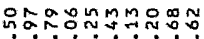

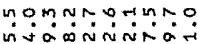

㱑文

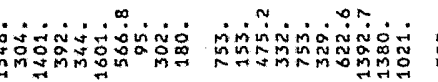

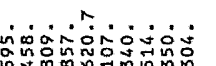

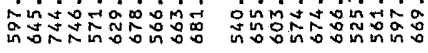

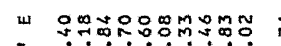

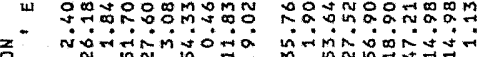

等

ำm

iñ⿻日禸

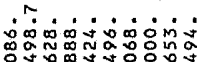

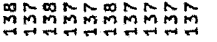

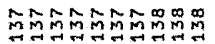

vioning

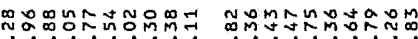

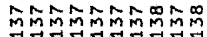

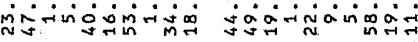

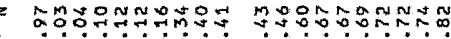

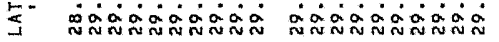

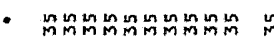

mº̛

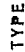

o

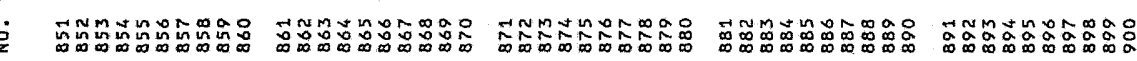

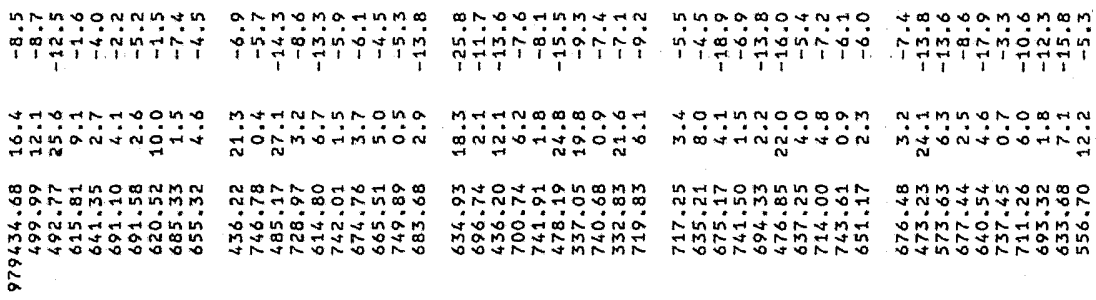

ming

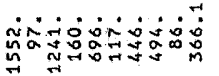

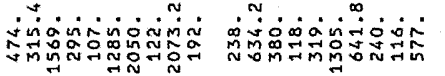

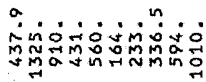

m

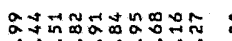

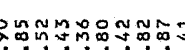

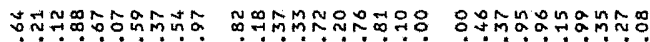

s.

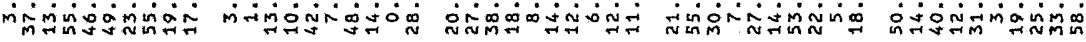

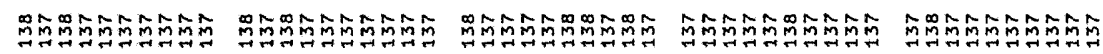

$z$

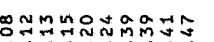

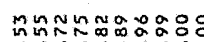

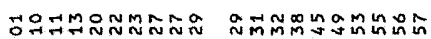

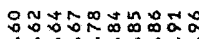

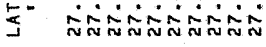

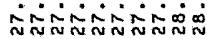

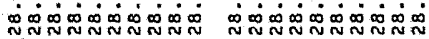

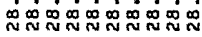

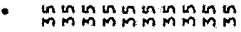

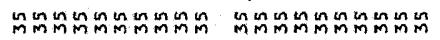

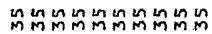

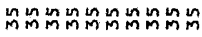

만 


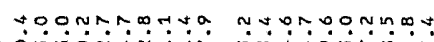

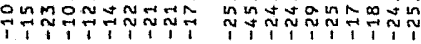

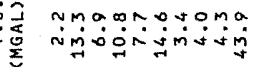

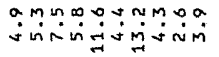

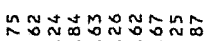

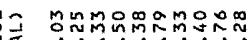
a â

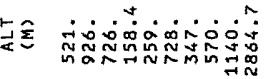

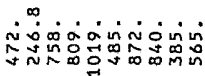

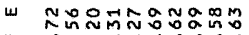

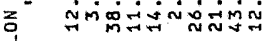

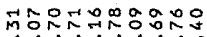

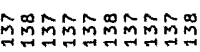

जिए

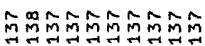

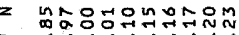

I लिiniminminimin

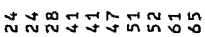

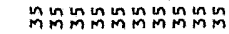

minmpinmpiniming

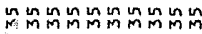

$\stackrel{w}{2}$

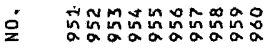

$\dot{2}$

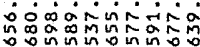

and

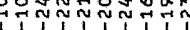

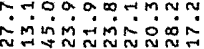

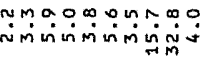

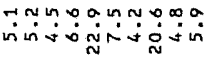

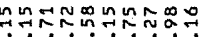

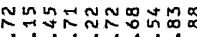

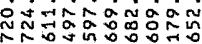

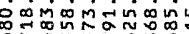

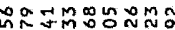

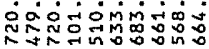

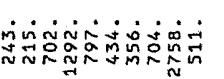

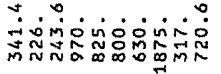

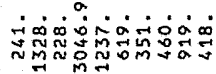

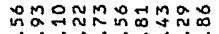

نที่ำ

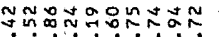

نंm

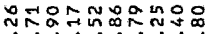

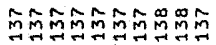

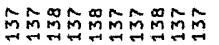

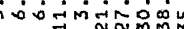

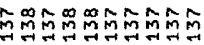

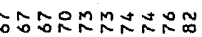

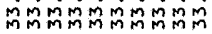

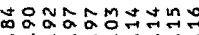

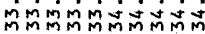

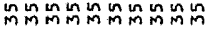

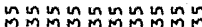

๓

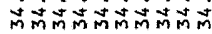

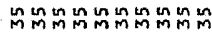

is ronomanom

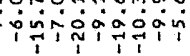

omorananaa

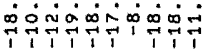

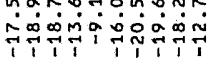

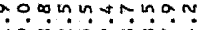

H HNN NOM

in ad

过

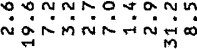

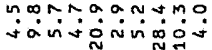

NNDMmOO

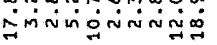

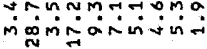

岂

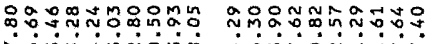

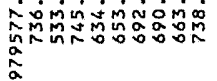

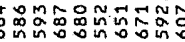

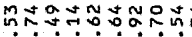

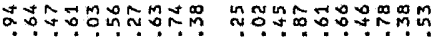

齿

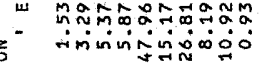

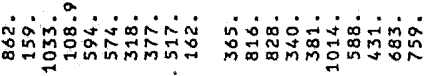

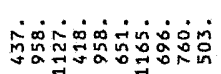

on

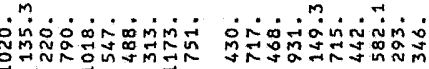

z

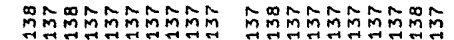

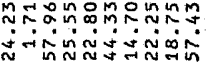

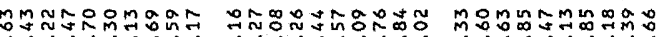
4ru出

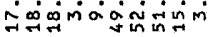

$z$

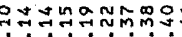

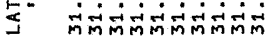
Y⿻ำ

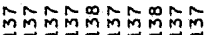

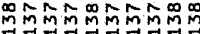

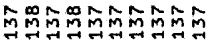

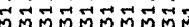

5oำn

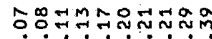

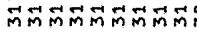

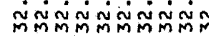

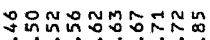

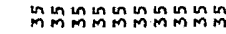

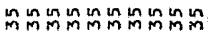

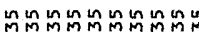

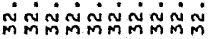

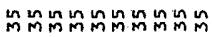

님

$\dot{2}$

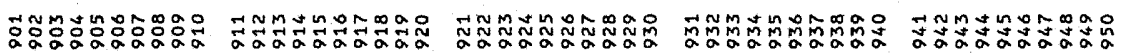




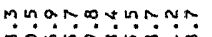

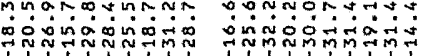

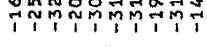

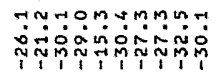

mogrmatinas

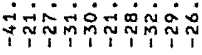

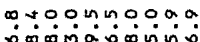

年

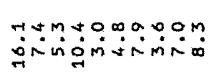

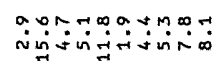

mosinming

ำ

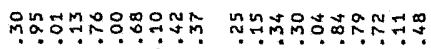

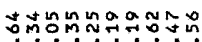

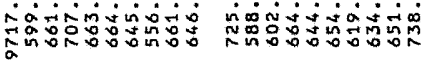

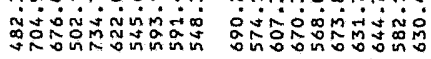

nim

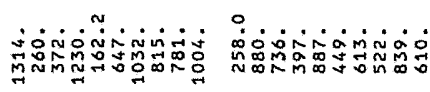

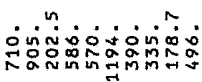

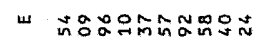

$z_{0}^{-}$minno

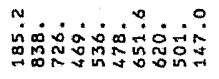

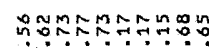

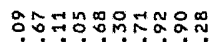

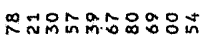

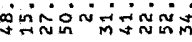

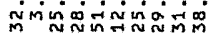

Ning

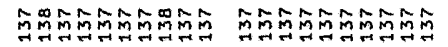

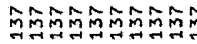

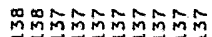

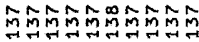

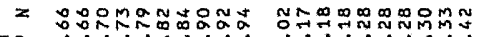

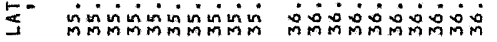

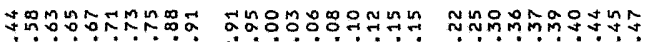

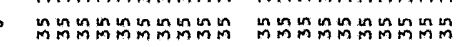

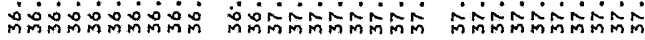

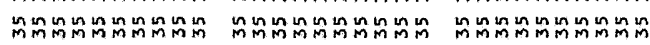

삘

$\stackrel{2}{2}$

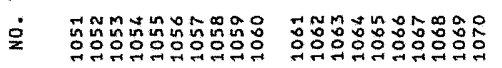

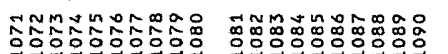

a h h

mrarinasinos

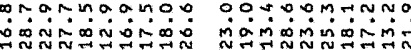

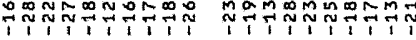

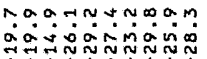

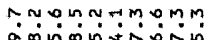

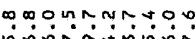

势

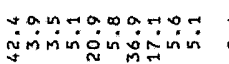

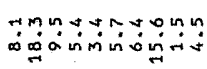

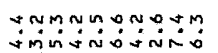

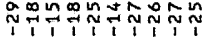

nom

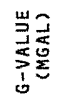

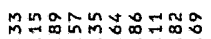

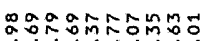

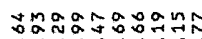

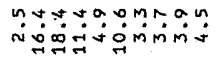

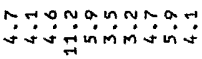

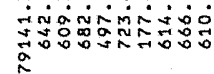

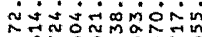

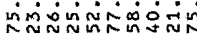

moñ

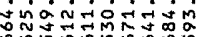

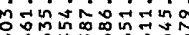

㔺

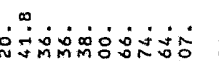

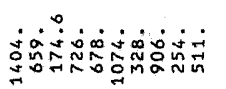

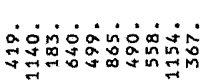

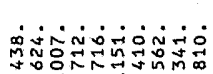

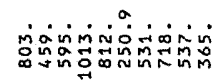

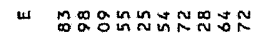

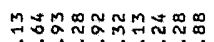

żo

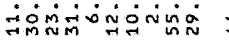

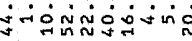

Кผต

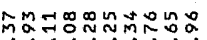

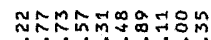

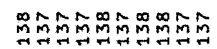

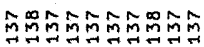

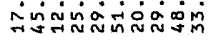

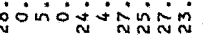

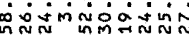

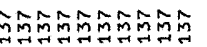

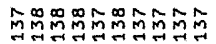

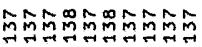

$z$ måñ

I

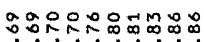

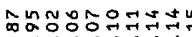

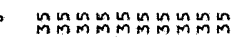

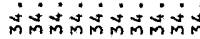

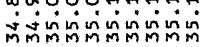

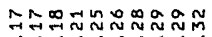

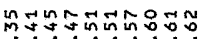

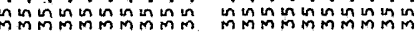

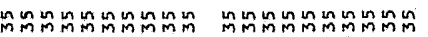

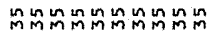

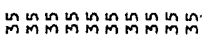

$\stackrel{\text { wa }}{2}$ ma

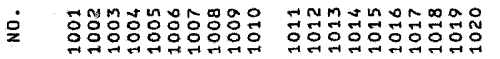

arado

a aad

$\alpha$ tana

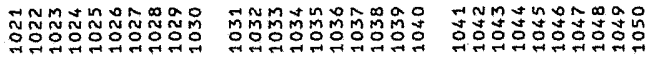




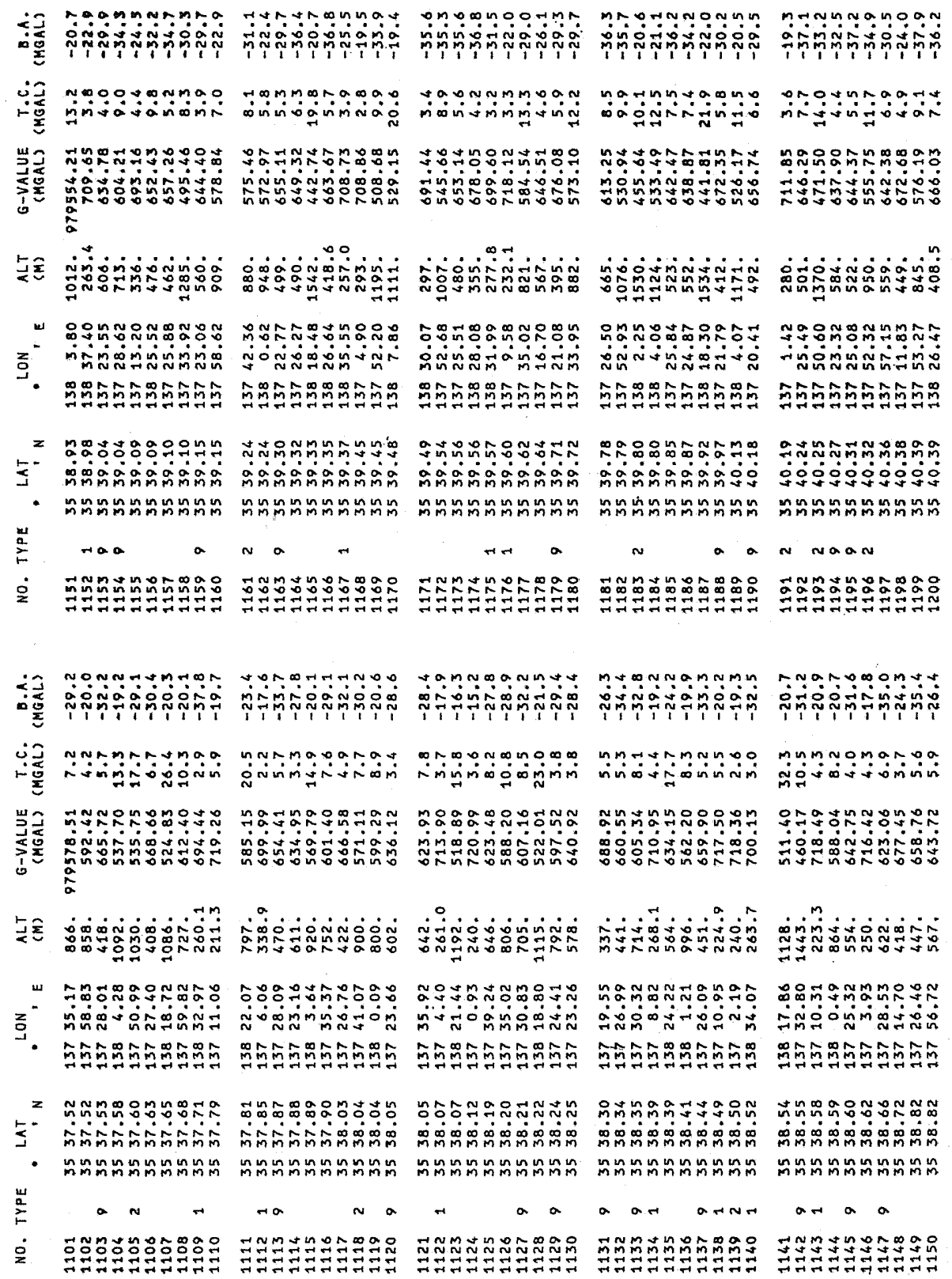




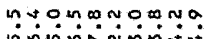

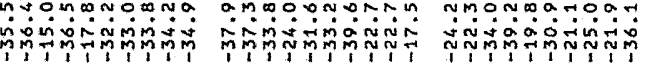

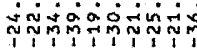

$0.04 \sin 200000$

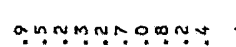

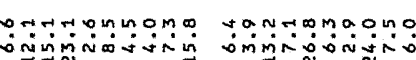

Trunannamos

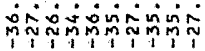

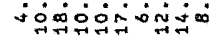

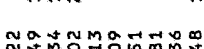

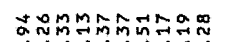

निव्न

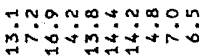

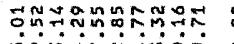

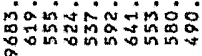

ainjo

กั่

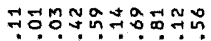

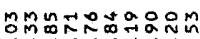
2

ñoำ $\circ$

rivisingas

t..................

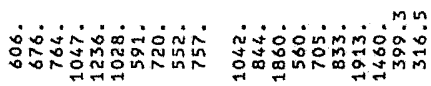

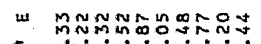

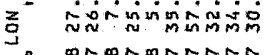

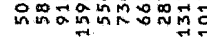

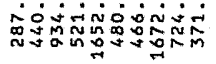

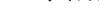

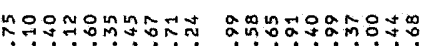

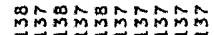

ñming

๓⿴囗十

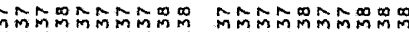

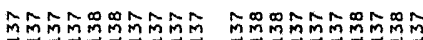

$z$ 下

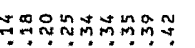

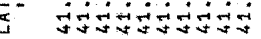

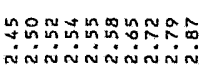

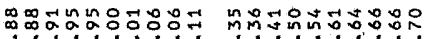

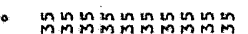

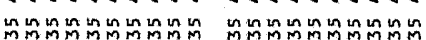

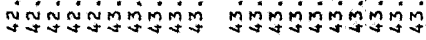

a

ma $N$

$\therefore a n$

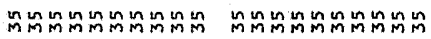

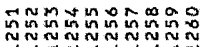

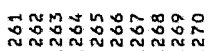

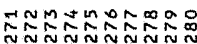

a a

$\sim a$

nad nan

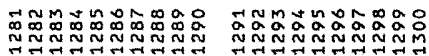

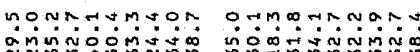

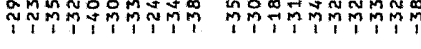

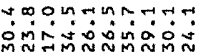

ㄴ.

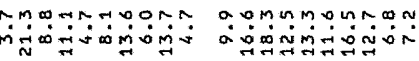

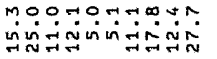

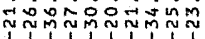

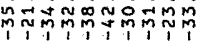

状变

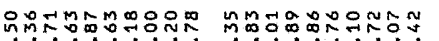

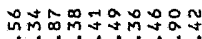

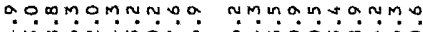

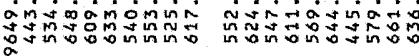

N

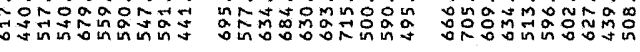

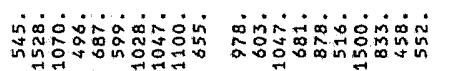

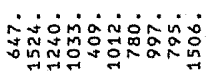

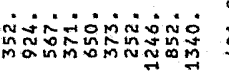

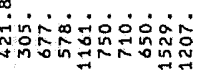

แ

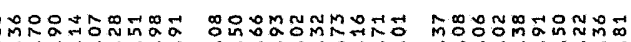

za

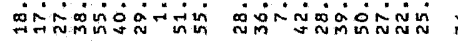

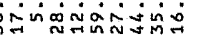

in

son

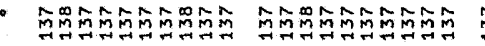

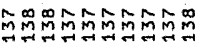

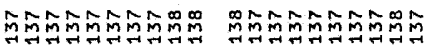

z ำำ

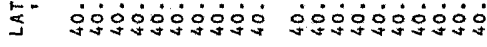

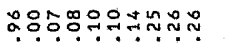

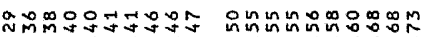

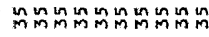

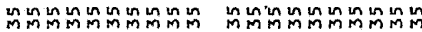

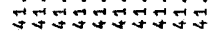

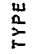
$\sim a a^{2}$

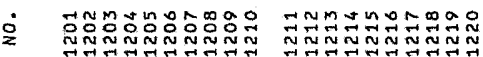

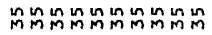




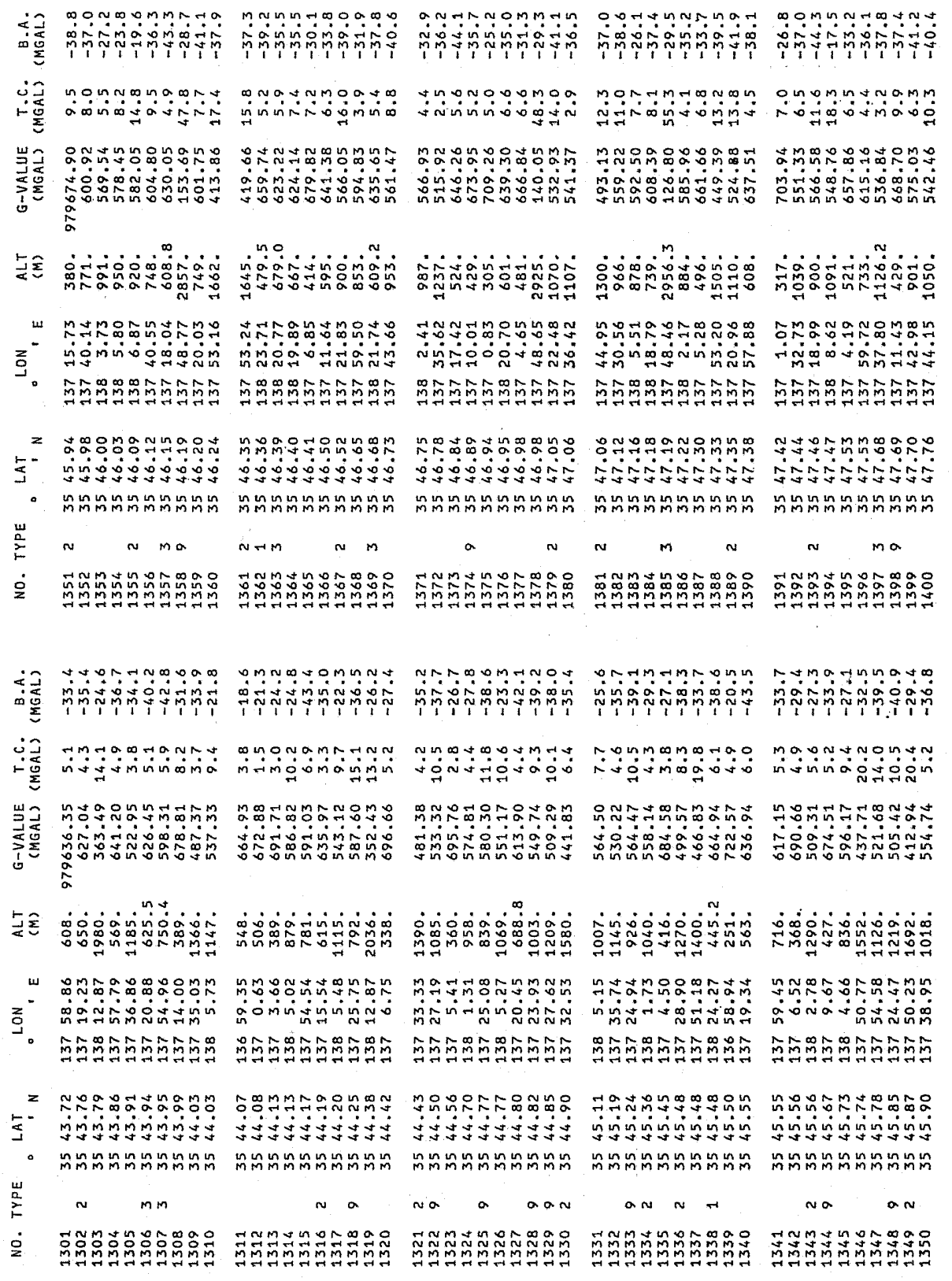




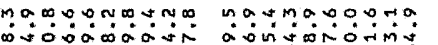
Yyiling

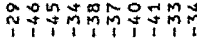

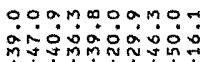

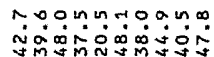

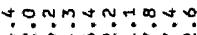
âting

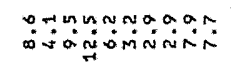

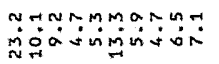

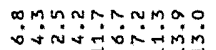

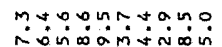

amanogonga

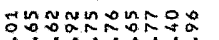

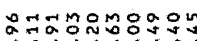

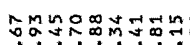

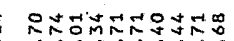

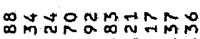

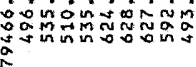

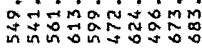

ofoninjing

sons

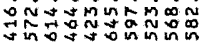

…m. ma

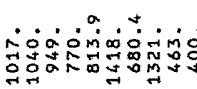

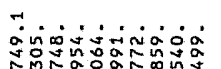

نำ

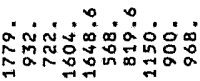

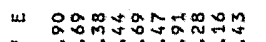

Z Niñañ

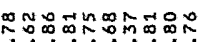

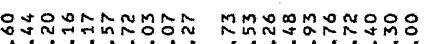

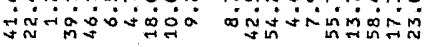

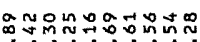

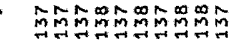

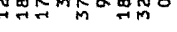

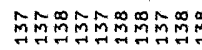

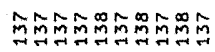

Sin $\min _{3}$

$z$

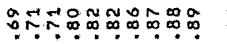

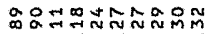

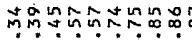

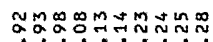

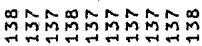

I gajagagojag

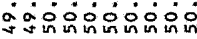
inio is i⿱宀

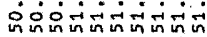

in

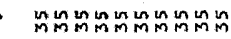

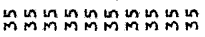

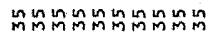

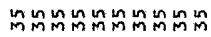

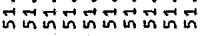

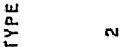

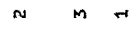

$\rightarrow \infty$

nen

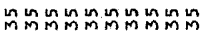

$\dot{0}$

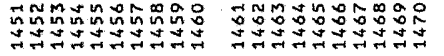

№n

$4 n$

$\sin$

nonoronana ơningming

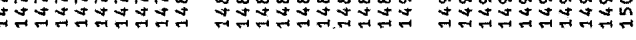

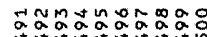

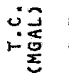

岁 0
$a$
4
0
0
0
$a$
$a$

占

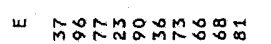

$z^{-}$ision

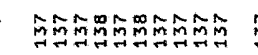

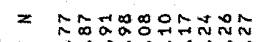

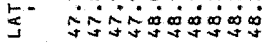

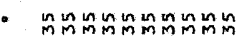

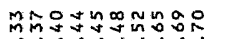

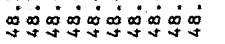

minnmminnm

a) NM

s

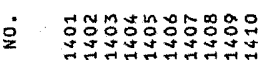

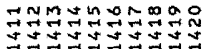

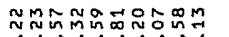

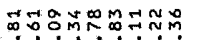

คำ

nOMNMNOOAa

Pơ

amooondoam oomninomnos ping

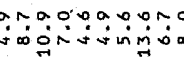

Momion

toin $\infty \mathrm{m} \infty$ in on

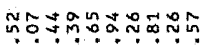

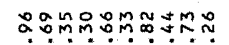

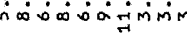

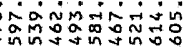

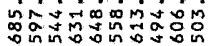

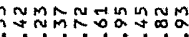

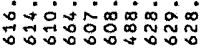

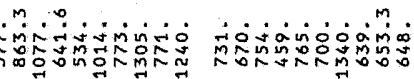

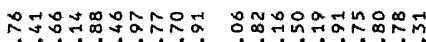

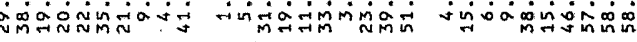

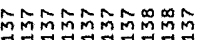

miñming

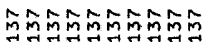

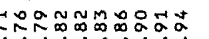

$\Rightarrow 00000$

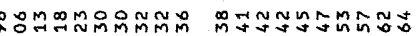

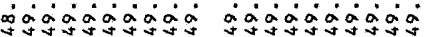

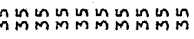

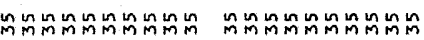

$m$

N $N$ N

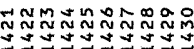

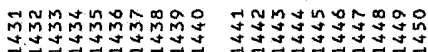


斿

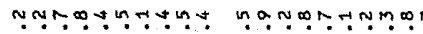

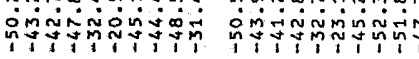

要

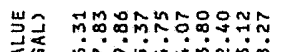

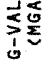

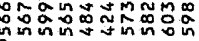
2

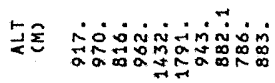

山 aูn

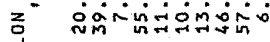

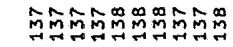

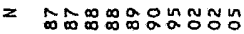

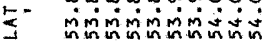

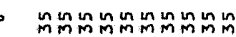

崖 N ํ.

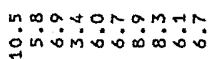

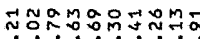

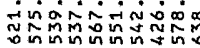

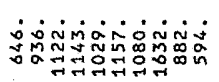

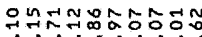

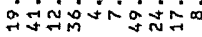

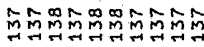

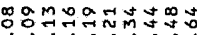

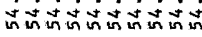

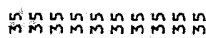

n

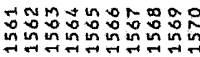

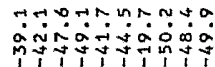

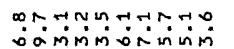

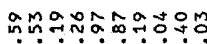

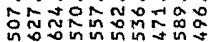

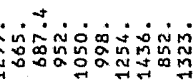

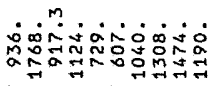

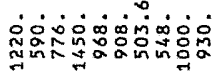

이에

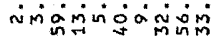

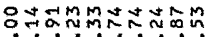

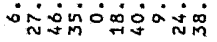

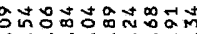

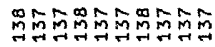

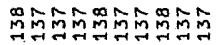

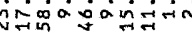

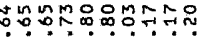

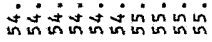

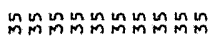

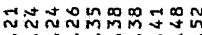

ถุ่ง

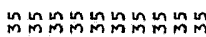

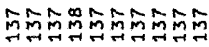

If Onintoonono

焉

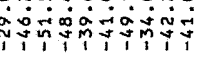

Nr.

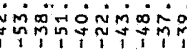

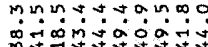

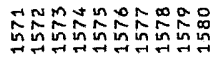

$\pi$

n $N$

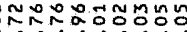

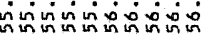

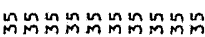

这

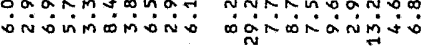

山己ํำ

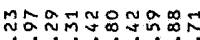

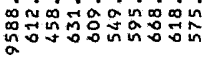

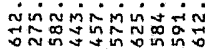
a

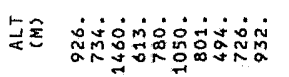

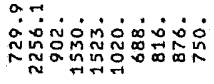

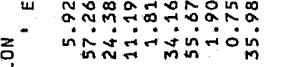

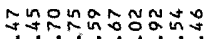

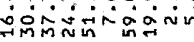

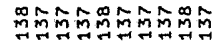

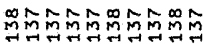

ن

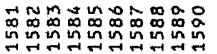

总

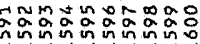

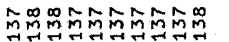

Dom num on m

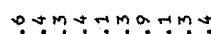

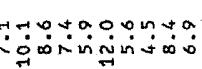

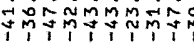

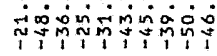

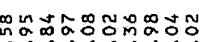

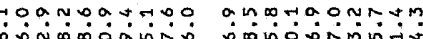

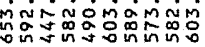

웅ํำ

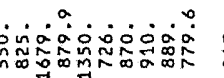

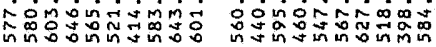

กู่

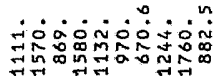

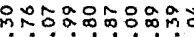

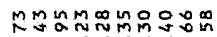

in

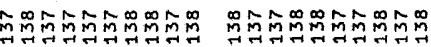

\footnotetext{
z 옹ำ

I in

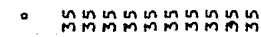

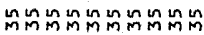

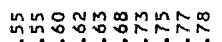

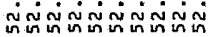

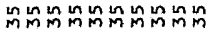

ํํㅇำ ผ่ंนี่

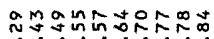

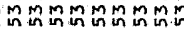

$\sim N$

Tim N

hN

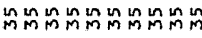

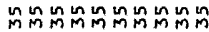

물

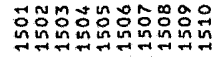

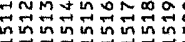

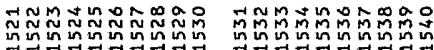

$N$

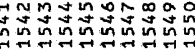


这

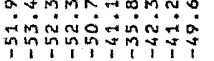

这全 mon

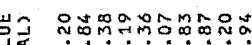

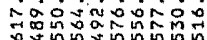

牙全

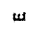

否

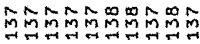

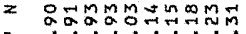

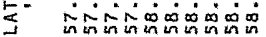

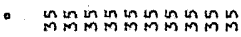

崖西

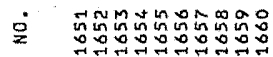

H.mpor

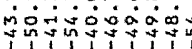

tomanum

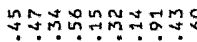

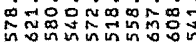

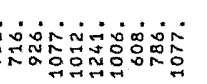

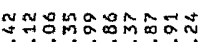

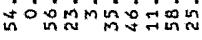

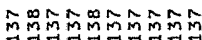

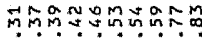

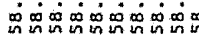

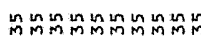

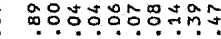

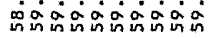

ñ?

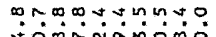

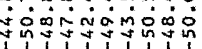

7nm: ing

nutomanoryn

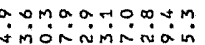

monunatonas

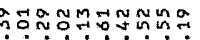

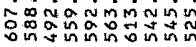

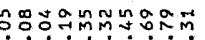

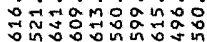

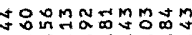

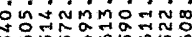

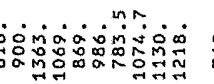

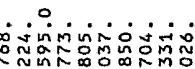

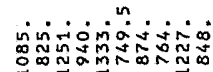

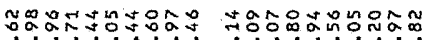

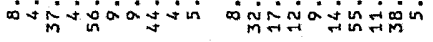

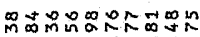

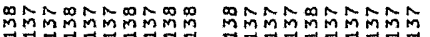

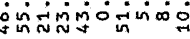

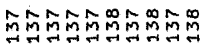

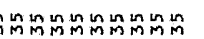

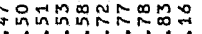
is is is is is is is is

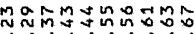

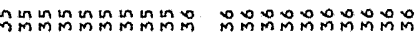

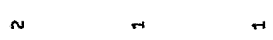

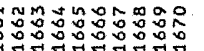

\section{每}

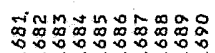

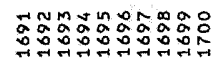

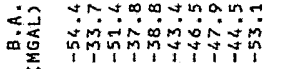

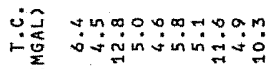

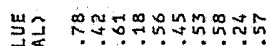

崖势

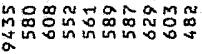

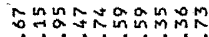

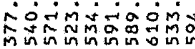
a

ヶ会

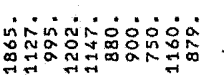

ân

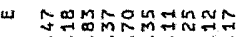

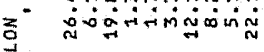

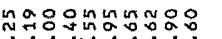

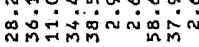

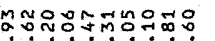

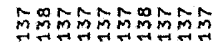

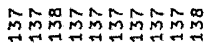

+agnoginivin

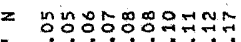

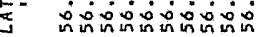

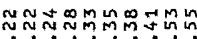

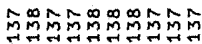

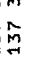

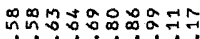

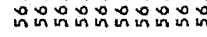

iñ

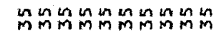

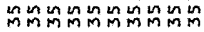

$n N$

岸 NNNA N

$\dot{8}$

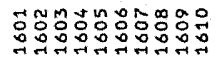

$n a$

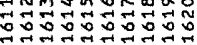

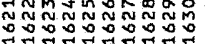

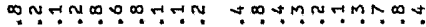

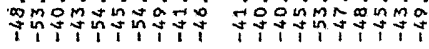

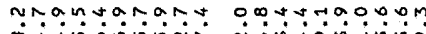

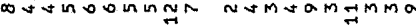

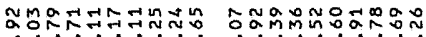

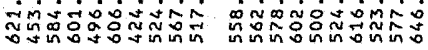

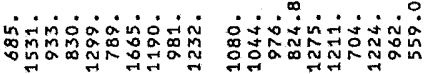

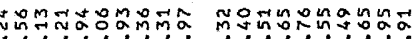

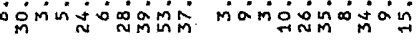

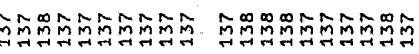

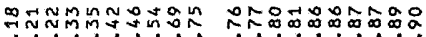

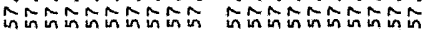

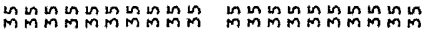

$\sim$

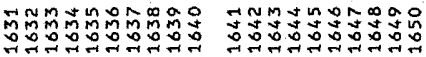




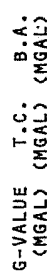
mand

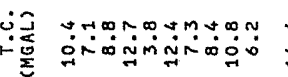

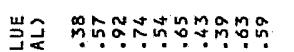

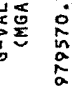

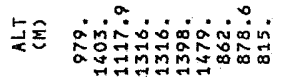

世 momporammo

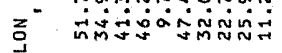

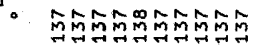

$z$ mơำ

- miminiminiminim

- 品品品品品品品星品品

$\stackrel{\text { un }}{2}$

官 $\operatorname{mos} 40.00 m a \infty$ pin

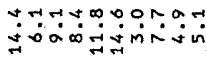

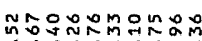

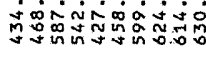

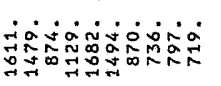

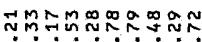

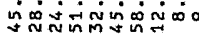

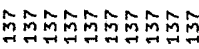

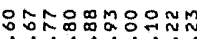

miminimintivis

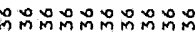

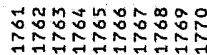

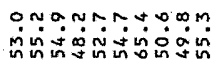

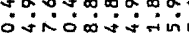

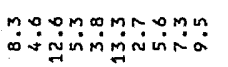

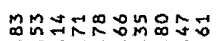

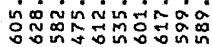

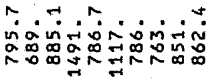

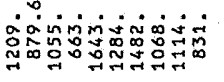

F⿻ำ

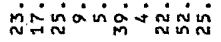

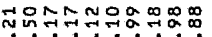

in نं

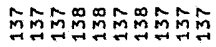

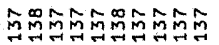

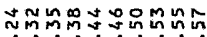

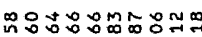

jutuquivinin

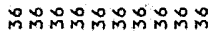

he $\mathrm{H}$

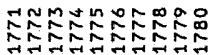

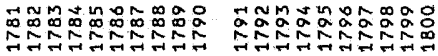

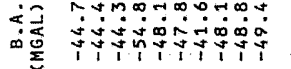

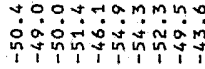
a
ancony

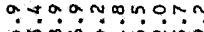

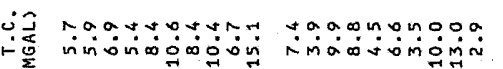

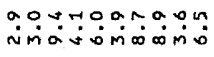

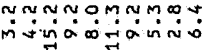

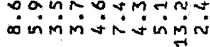

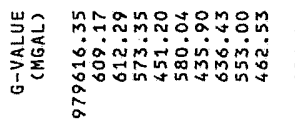

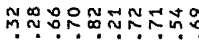

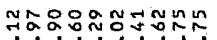

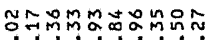

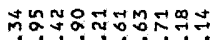

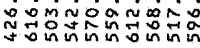

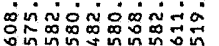

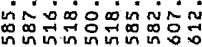

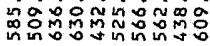

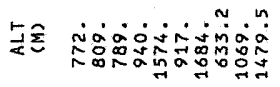

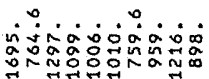

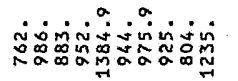

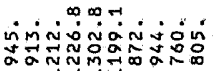
a.

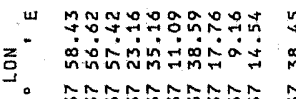

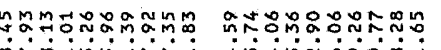

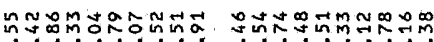 命解解解解商
ñoññmon

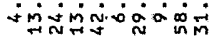

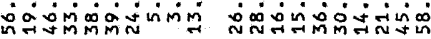

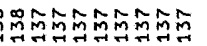

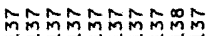

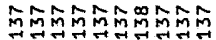

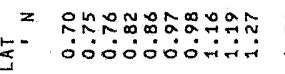

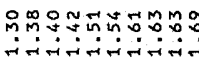

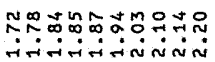
Nmiñ

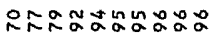$$
\text { . }
$$
- 品品品品品品品品品
罂

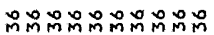

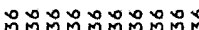
लंniniviviviñ

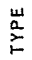
$\dot{2}$

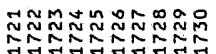

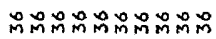




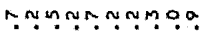

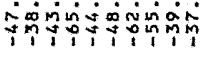

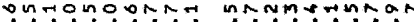

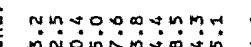

op? Tragmon ipinipicin

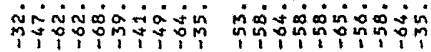

ân

iminiviningo

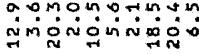

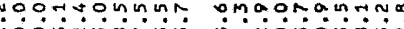

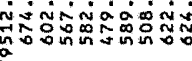

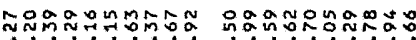

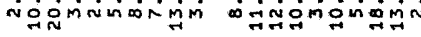
ล

คำ

mơ

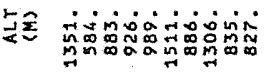

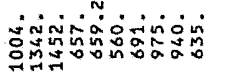

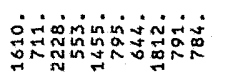

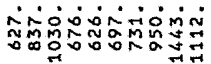

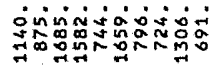

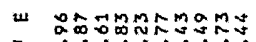

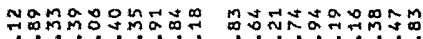

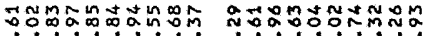

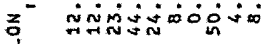

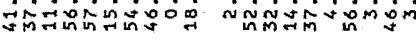

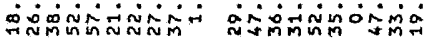

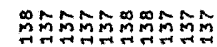

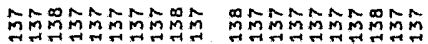

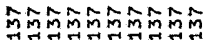

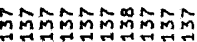

z

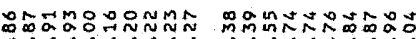

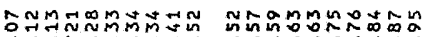

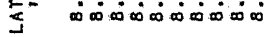

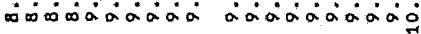

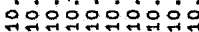

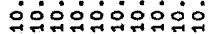

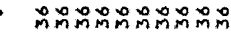

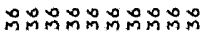

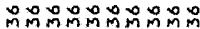

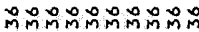

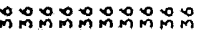

ia

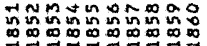

Find

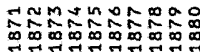

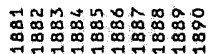

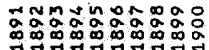

(3)

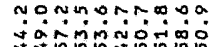

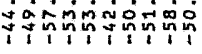

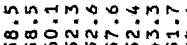

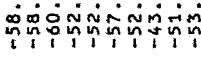

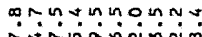

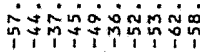

बmon

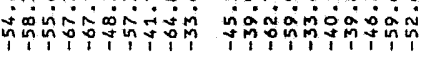

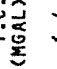

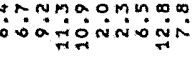

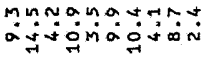

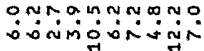

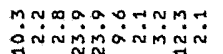

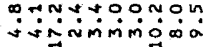

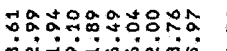

miñ

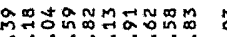

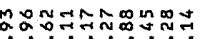

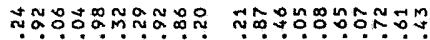
a

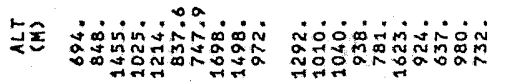

मु०

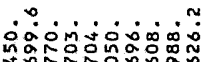

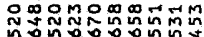

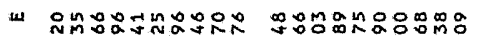

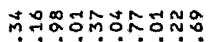

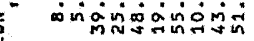

N

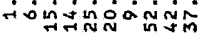

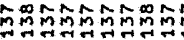

Doñ

moñ

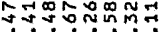

in inmminiog

Diñ

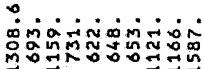

= ํㅜㅇำกำ

s-

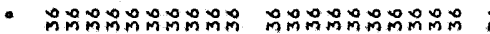

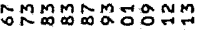

繀

C

i

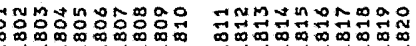

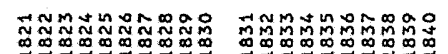

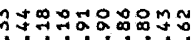

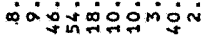

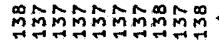
ritrivition on

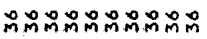

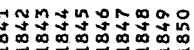




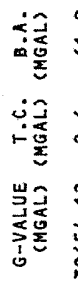

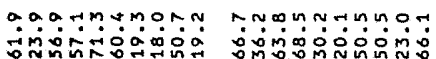

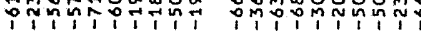

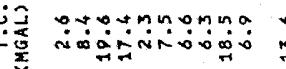

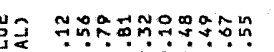

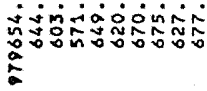

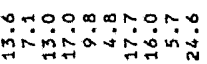

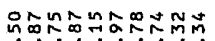
ingining

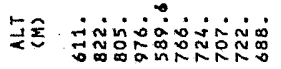

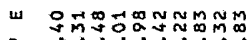

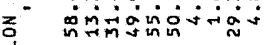

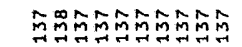

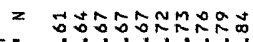

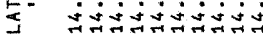

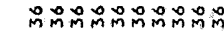

w

$\dot{\rho}$

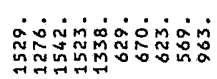

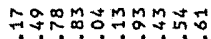

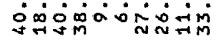

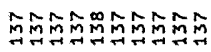

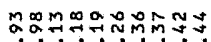

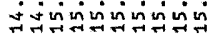

กำกำกำกำกำกำกำ

a

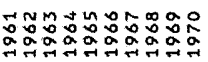

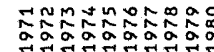

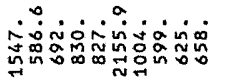

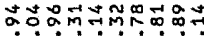

gingminim

解解解的解

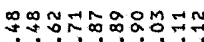

ที丶n

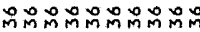

กับกำกำษ

नं०

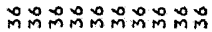

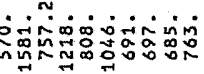

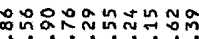

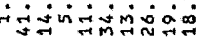

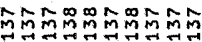

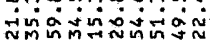

解鳝解解解

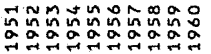

通

nomonnasono

:

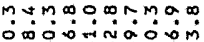

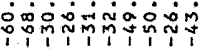

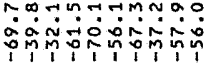

高

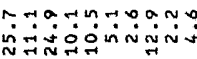

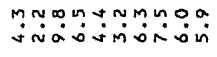

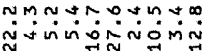

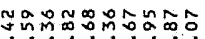

닌손

우ำํำ?

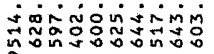

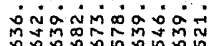

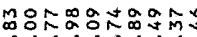

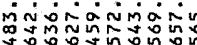
a

去

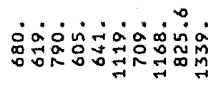

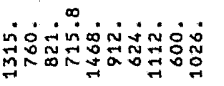

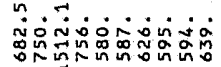

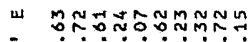

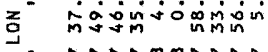

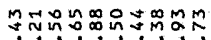

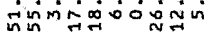

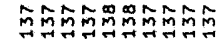

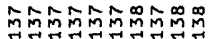

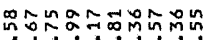

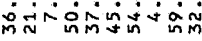

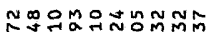

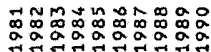

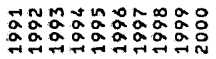

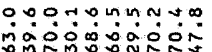

ipitiviti

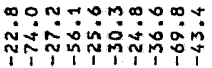

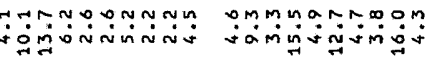

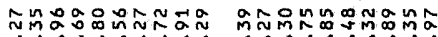

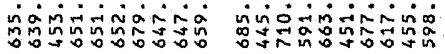

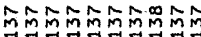

年

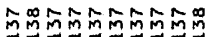

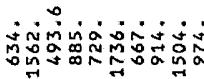

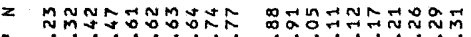

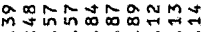

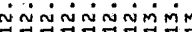

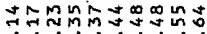

mimmingmimming

ํํํํํㅇํํㅇํㅇ

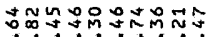

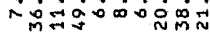

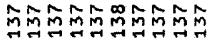

-

ตํํํํํํํํำ品

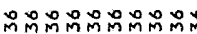

NNM

ลำ $m \rightarrow \infty$

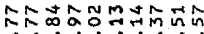

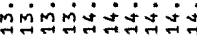

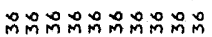

$4 \pi$

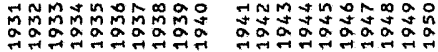




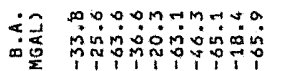

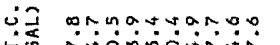

造

岂列

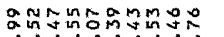

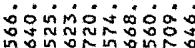
a

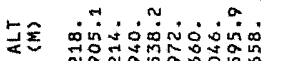

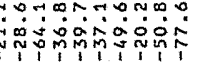

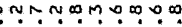

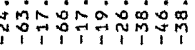

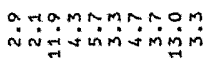

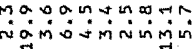

m.40ำ

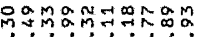

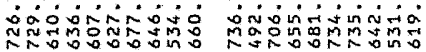

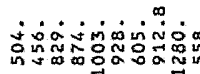

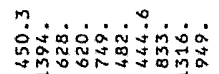

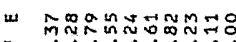

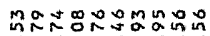

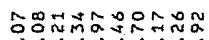

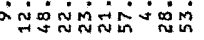

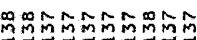

तु

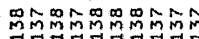

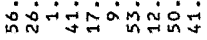

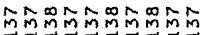

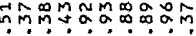

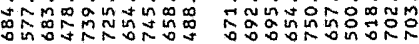

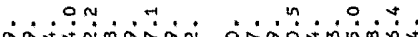

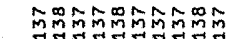

z DOM응유NNN

5-

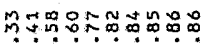

romang

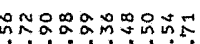

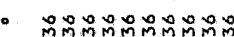

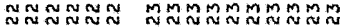

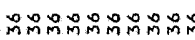

iminimind

ํํํํํํํํํํํํํํํำ

in

览

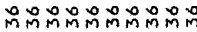

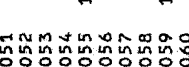

等

$\dot{x} \dot{\alpha} \dot{\alpha} \dot{\alpha} \dot{\alpha} \dot{\alpha} \dot{\alpha}$

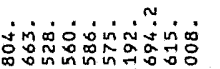

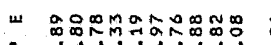

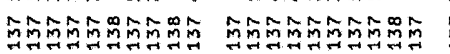

arogonanging

웅ำ

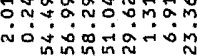

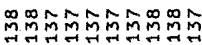

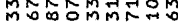

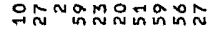

$z$

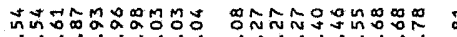

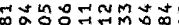

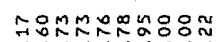

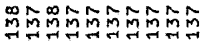

5

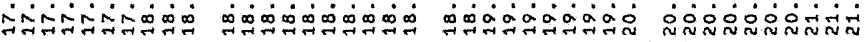

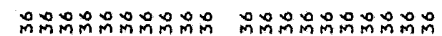

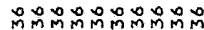

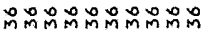

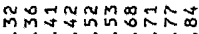

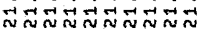

$\frac{w}{2}$

定 


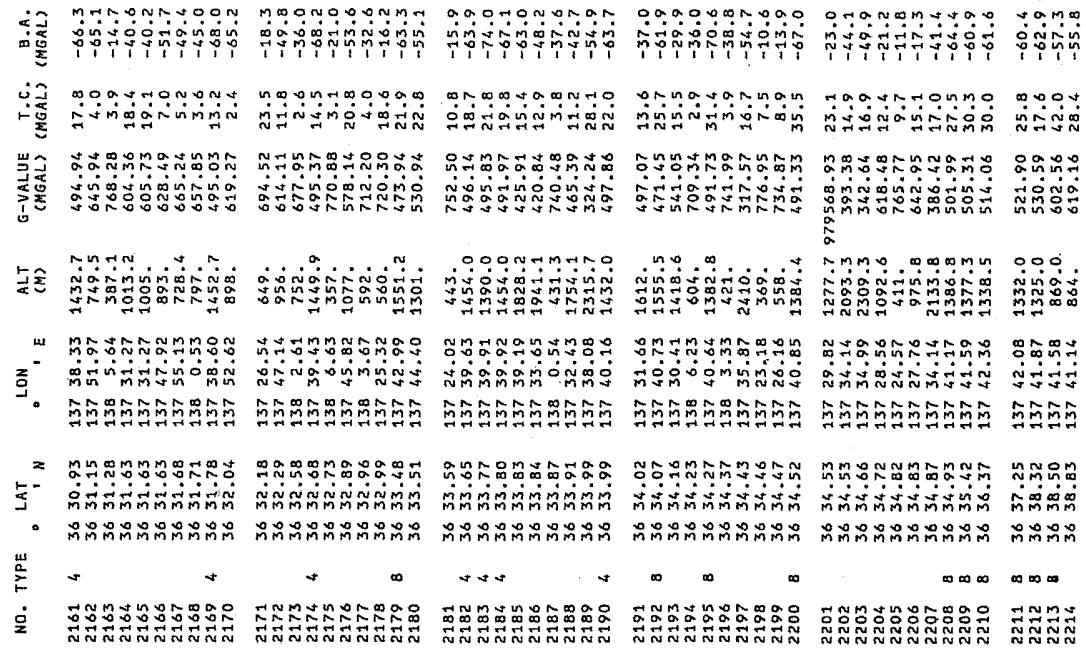

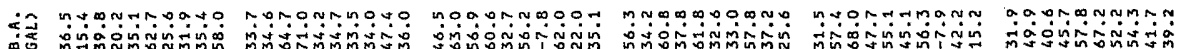

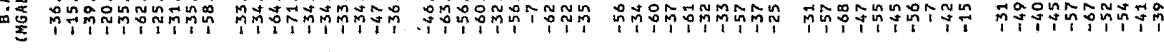

ب.

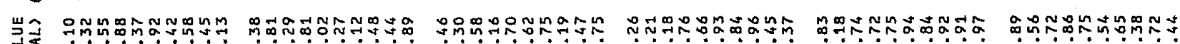

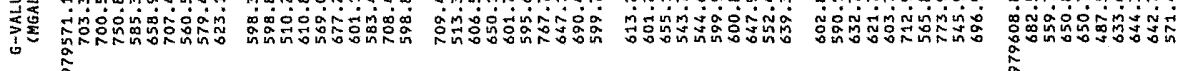

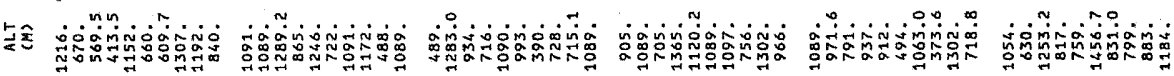

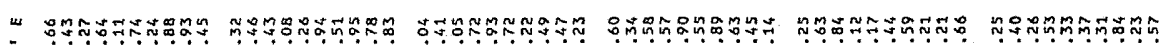

z"

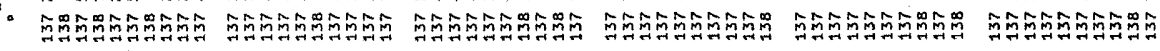

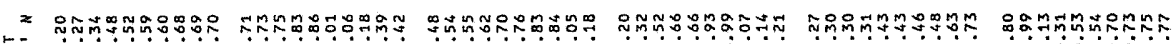

×

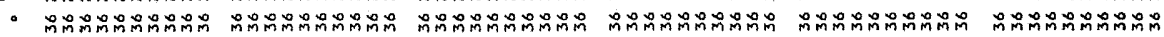

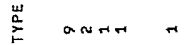

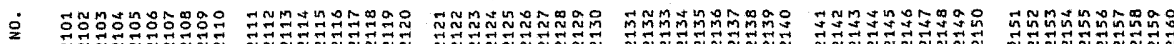

